\title{
Effects of multi-scale and regular grid geometries on decaying turbulence
}

\author{
R. Jason Hearst ${ }^{1,2}$ and Philippe Lavoie $^{1} \dagger$ \\ ${ }^{1}$ Institute for Aerospace Studies, University of Toronto, Toronto, ON M3H 5T6, Canada \\ ${ }^{2}$ Engineering and the Environment, University of Southampton, Southampton, SO17 1BJ, \\ United Kingdom
}

(Received xx; revised xx; accepted xx)

The influence of a multi-scale fractal-based geometry on the decay of turbulence is investigated by comparing the turbulence produced by a square-fractal-element grid to that produced by two regular grids with similar physical properties. Comparison of the grid wakes at constant grid Reynolds number, $R e_{M}$, identifies that in the farfield both regular grids produce comparable or higher turbulence intensities and local Reynolds numbers, $R e_{\lambda}$, than the square-fractal-element grid. This result is illustrative of a limitation of multi-scale geometries to produce the oft-quoted high levels of turbulence intensity and $R e_{\lambda}$. In the far-field, the spectra are approximately collapsed at all scales for all three grids at a given $R e_{\lambda}$. When a non-equilibrium near-field spectrum with $\langle u v\rangle \neq 0$ is compared to a far-field spectrum at the same $R e_{\lambda}$ but with $\langle u v\rangle \approx 0$, it is shown that their shapes are markedly different and that the non-equilibrium spectrum has a steeper slope, giving the appearance of being nearer $k^{-5 / 3}$, although there is no theoretical expectation of an inertial range at such locations in the flow. However, when a non-equilibrium spectrum with $\langle u v\rangle \approx 0$ is compared to a far-field spectrum at the same $R e_{\lambda}$, they are once again collapsed. This is shown to be related to non-zero Reynolds shear stress at scales that penetrate the scaling range for the present experiment, and hence the influence of shear is not limited to the largest scales. These results demonstrate the importance of local properties of the flow on the turbulence spectra at given locations in the inherently inhomogeneous flow found in the non-equilibrium region downstream of grids. In particular, how the presence of local shear stress can fundamentally change the shape of the spectra at scales that can be mistakenly interpreted as an inertial range.

Key words: (to be entered by typesetter)

\section{Introduction}

The advent of fractal-generated multi-scale turbulent flows has allowed new and innovative questions to be posed of our understanding of the turbulent motion of fluids; see for instance the review by Vassilicos (2015). Fractal-generated flows also open the door for several novel applications of multi-scale turbulence generators, e.g., low-noise aerodynamic spoilers (Nedić et al. 2012) and enhanced mixing for combustion applications (Soulopoulus et al. 2013; Verbeek et al. 2015). Practically, fractal-generated flows are attractive because they have been reported to produce turbulence with higher turbulence intensities and local Reynolds numbers than regular grids with similar blockage, $\sigma$, and

$\dagger$ Email address for correspondence: lavoie@utias.utoronto.ca 
pressure drops (Hurst \& Vassilicos 2007; Geipel et al. 2010; Valente \& Vassilicos 2011; Kinzel et al. 2011; Laizet \& Vassilicos 2015). Theoretically, fractal-generated flows have garnered interest because they produce a region of rapid energy decay that exhibits a long (two decade) power-law range in the spectra that closely matches $k^{-5 / 3}$ (Mazellier \& Vassilicos 2010), but that has non-constant normalised dissipation scaling (Valente \& Vassilicos 2011; Hearst \& Lavoie 2014a; Vassilicos 2015). This appears to be at odds with our classical understanding of the dynamics of turbulence. Flow with similar phenomenology has also been observed behind regular grids (Valente \& Vassilicos 2012; Isaza et al. 2014). Such turbulence has been termed 'non-equilibrium' turbulence, and is a topic of intense contemporary research with far reaching consequences for others flows, e.g., wakes (Nedić et al. 2013; Dairay et al. 2015; Castro 2016), and jets (Geipel et al. 2010; Cafiero et al. 2015).

The wake of square fractal grids has been an area of particular interest in recent years because they were shown to produce turbulence that was markedly different from regular grids and fractal cross-grids (Hurst \& Vassilicos 2007; Valente \& Vassilicos 2011). For instance, square fractal grids produce an extended non-equilibrium region (relative to regular grids) that experiences rapid decay of the turbulent kinetic energy (Hurst \& Vassilicos 2007; Valente \& Vassilicos 2011; Hearst \& Lavoie 2014a). The decay of the energy in this region follows a power-law, $\left\langle q^{2}\right\rangle \sim\left(x-x_{0}\right)^{n}$, with $n \lesssim-2.5$ (Valente \& Vassilicos 2011; Hearst \& Lavoie 2014a); where $\left\langle q^{2}\right\rangle=\left\langle u^{2}\right\rangle+\left\langle v^{2}\right\rangle+\left\langle w^{2}\right\rangle$ is twice the turbulent kinetic energy, $x$ is the downstream distance from the grid, and $x_{0}$ is a virtual origin. However, canonical decay rates range $-1 \geqslant n \geqslant-1.4$, which are substantially slower (Comte-Bellot \& Corrsin 1966; Mohamed \& LaRue 1990; Lavoie et al. 2007; Krogstad \& Davidson 2010, 2011). Despite the rapid decay of energy in the non-equilibrium region, it is the region with the highest turbulence intensities and Taylor microscale Reynolds numbers. The Taylor microscale Reynolds number is given by,

$$
R e_{\lambda}=\frac{\left\langle u^{2}\right\rangle^{1 / 2} \lambda}{\nu}
$$

where the Taylor microscale is,

$$
\lambda^{2}=15 \nu \frac{\left\langle u^{2}\right\rangle}{\langle\epsilon\rangle},
$$

and $\langle\epsilon\rangle$ is the mean turbulent kinetic energy dissipation rate (hereafter referred to simply as dissipation). The assertion that square fractals produce higher turbulence intensities and $R e_{\lambda}$ than regular grids has largely been based on comparisons where a single square fractal occupied the entire wind tunnel cross-section and the mesh length, $M$, of the regular grid was significantly smaller than the maximum length of an element in the fractal geometry, $L_{0}$, i.e., $L_{0} \gg M$ (Hurst \& Vassilicos 2007; Laizet \& Vassilicos 2015); for a regular grid, $L_{0}=M$. In the few studies where $L_{0}$ of the fractal and $M$ of the regular grid were comparable, the regular grid configuration included only four bars (Valente \& Vassilicos 2012, 2014; Zhou et al. 2014; Laizet et al. 2015) and it is quite possible the interaction of the bar wakes with the domain edges/walls influenced the development of the flow (Wang \& George 2002). Moreover, all such comparisons have been made in the region $x / L_{0}<20$, where the fractals experience non-equilibrium phenomenology (Hearst \& Lavoie 2014a), while the regular grids have already begun to transition to a canonical grid turbulence far-field (Isaza et al. 2014). In an unconfined environment, the turbulence in the wake of multi-scale and regular grids would continue to evolve laterally as well as in the streamwise direction, and there has not been a rigorous comparison of the far-field of regular grids and square fractal grids where $L_{0}=M$ and $\sigma$ has been matched. Indeed, 
one might expect that given the extended region of rapid decay behind a fractal relative to a regular grid, the far-field might be less energetic. However, there is no information in the literature that has identified or inferred that this is the case. This point is important for applications where grids are used to produce enhanced turbulence, either locally or for an extended region. Investigating this further is the first focus of the present work.

The second focus is centred about the the shape of the spectra in the scaling range. Here, the term scaling range is used to describe the subset of wavenumbers where the velocity spectrum scales with a power-law, $k^{m}$, where $m$ is a constant. The scaling range is the inertial range predicted by Kolmogorov-based analysis when $k^{-5 / 3}$. For both regular and multi-scale grids, it would appear that spectra in the non-equilibrium region approach an apparent $k^{-5 / 3}$ region at lower $R e_{\lambda}$ than equivalent spectra in the far-field (Mazellier \& Vassilicos 2010; Valente \& Vassilicos 2011, 2012; Hearst \& Lavoie 2014b; Isaza et al. 2014; Laizet et al. 2015). The presence of a near $k^{-5 / 3}$ spectrum in the non-equilibrium region is interesting because the non-equilibrium region experiences non-constant normalised dissipation scaling (Valente \& Vassilicos 2011, 2014). Constant normalised dissipation scaling and $k^{-5 / 3}$ are thought to be results of the same phenomenology as they are classically derived from the same base set of assumptions (Lumley 1992). Interestingly, recent studies have observed turbulent transport of kinetic energy and turbulent production in the region that experiences a near $k^{-5 / 3}$ spectrum (Valente \& Vassilicos 2011, 2014; Nagata et al. 2013; Hearst \& Lavoie 2014b). This is also curious because Kolmogorov-based theory is meant to apply for turbulence that is sufficiently removed from its boundary and initial generating conditions to be uninfluenced by them (Kolmogorov 1941; Frisch 1995; Isaza et al. 2014). These near $k^{-5 / 3}$ spectra have typically been reported along a single axis of the flow, and hence it is unclear if these observations are a consequence of a global phenomenological difference between the non-equilibrium region and the far-field, or whether they are a consequence of local properties present at very specific locations in the flow field. For instance, observations of near $k^{-5 / 3}$ spectra have also been reported for the highly sheared regions of a turbulent boundary layer (Saddoughi \& Veeravalli 1994), and jets (Mi \& Antonia 2001). Laizet et al. (2015) conducted an extensive study along the centreline of the wakes of space-filling square fractal and four-bar regular grids to demonstrate that a $f^{-5 / 3}$ range in the frequency spectrum originates in the production region that precedes the peak in the turbulence intensity. They associated this range with intermittent clusters of elongated vortices prevalent near the grid. They also noted that the $f^{-5 / 3}$ region should not be confused with a Kolmogorov $k^{-5 / 3}$ spectrum because their results did not collapse when normalised by the dissipative Kolmogorov scaling. A common theme amongst these observations of near $k^{-5 / 3}$ spectra is the presence of Reynolds shear stress, $\langle u v\rangle$, which is also featured in the production term (Nagata et al. 2013; Valente \& Vassilicos 2014; Hearst \& Lavoie 2014b; Isaza et al. 2014). This may be a statistical marker of the vortex clusters identified by Laizet et al. (2015). Perhaps the near $k^{-5 / 3}$ spectra at the relatively low $R e_{\lambda}$ of grid turbulence are only present at locations where there is $\langle u v\rangle$, similar to observations in turbulent boundary layers and jets. Determining this requires more detailed measurements of the non-equilibrium region including both positions where $\langle u v\rangle \neq 0$ and $\langle u v\rangle=0$ with direct comparison to the far-field at similar $R e_{\lambda}$. Thus, clarifying the conditions that generate a spectrum that appears to have a $k^{-5 / 3}$ range where one is not expected is the second focus of this work.

To address the above issues, we investigate the wake of a square-fractal-element grid and compare it to the wakes of two different regular grids with the same blockage. Comparisons are made of the evolution of the turbulence intensity and $R e_{\lambda}$ at constant $R e_{M}=U_{0} M / \nu$ in order to ascertain how long the desirable characteristics of fractal- 


\begin{tabular}{cccccccccccl} 
Grid & $L_{0}=M$ & $L_{1}$ & $L_{2}$ & $L_{3}$ & $\tau_{0}$ & $\tau_{1}$ & $\tau_{2}$ & $\tau_{3}$ & $\sigma$ & $x^{*}$ & Type \\
\hline Fs39 & 100.0 & 55.6 & 24.7 & 11.0 & 6.7 & 4.1 & 2.5 & 1.5 & 0.39 & 1493 & fractal-element \\
$S q 39$ & 100.0 & - & - & - & 22.0 & - & - & - & 0.39 & 455 & planar regular \\
$R d 38$ & 32.0 & - & - & - & 6.8 & - & - & - & 0.38 & 151 & bi-planar round-rod
\end{tabular}

TABLE 1. Grid dimensions and properties; lengths and thicknesses are given in mm. For Fs39, $L_{i}$, and $\tau_{i}$, with $i=0,1,2,3$, represent the length and the thickness, respectively, of the elements in the square-fractal-element grid from largest to smallest.

generated flows persist relative to the regular grids; $U_{0}$ is the velocity immediately upstream of the grid. Comparisons are also made between positions in the non-equilibrium region and in the far-field at constant $R e_{\lambda}$. The purpose of the $R e_{\lambda}$ matched survey is to determine: (i) if the spectra indeed are closer to $k^{-5 / 3}$ in the non-equilibrium region, (ii) what characteristics the flow possesses at locations where a near $k^{-5 / 3}$ spectrum is observed, and (iii) if near $k^{-5 / 3}$ spectra are ubiquitous across the non-equilibrium region.

\section{Experimental procedure}

\subsection{The grids}

The first grid is the square-fractal-element grid, Fs39, used by Hearst \& Lavoie $(2014 a, b, 2015 b)$. This grid consists of several $N=3$ square-fractal-elements mounted to a $12 \times 8$ background mesh (where $N$ is the number of times the fractal shape is repeated). This grid includes multiple prefractal elements across the test-section, allowing for measurements to be made without influence from the walls, and farther downstream relative to the largest element in the grid, $L_{0}$. Throughout this work, we define the mesh length of a grid as $M=L_{0}$; Hearst \& Lavoie (2014a) showed that this was a relevant choice based on the far-field collapse of normalised dissipation for Fs39 with regular grids. Fs39 was laser cut from a single piece of $3 \mathrm{~mm}$ thick stainless steel. The exact dimensions of the square-fractal-element grid are provided in table 1.

Two canonical 'regular' grids were also used as benchmarks. The first is a standard square mesh, $S q 39$, with $M=100 \mathrm{~mm}$ such that $M$ is the same for both $S q 39$ and $F s 39$. The solidity, $\sigma$, of $S q 39$ and $F s 39$ was also designed to be the same. $S q 39$ was water jet cut from a single piece of $6.35 \mathrm{~mm}$ thick aluminum. The last grid is $R d 38$, which is a classic bi-planar round rod grid. The grid is composed of aluminum round rods with a $6.8 \mathrm{~mm}$ nominal diameter, mounted into a machined frame with $M=32 \mathrm{~mm}$. The maximum bar thickness, $\tau_{0}$, and the blockage, $\sigma$, of $R d 38$ and Fs39 are nearly equal. Dimensions for all three grids are provided in table 1, and images of a sample area of each grid are shown in figure 1 . The 'wake interaction length scale' as defined by Mazellier \& Vassilicos (2010), $x^{*}=L_{0}^{2} / \tau_{0}$, is also provided in table 1 for reference.

\subsection{Experimental facility}

All measurements were performed in the low-speed, recirculating wind tunnel at the University of Toronto Institute for Aerospace Studies. A schematic of the tunnel is provided in figure 2. The wind tunnel is powered by a 60 h.p. 3-phase motor. The motor is situated inside the wind tunnel and the motor housing section is isolated from the rest of the wind tunnel by soft connections that reduce the transmission of mechanical vibrations. Without a grid, a mean flow velocity of $40 \mathrm{~m} / \mathrm{s}$ is achievable and the turbulence 


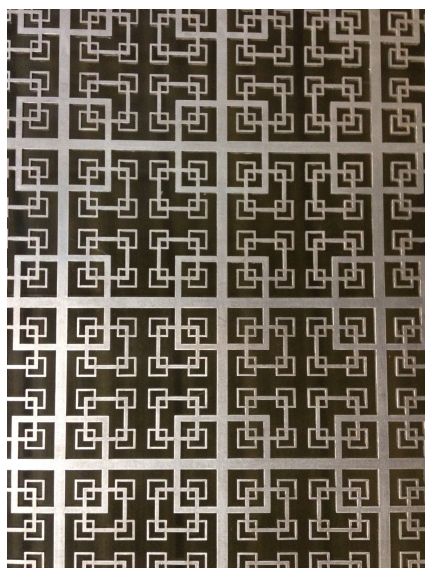

(a)

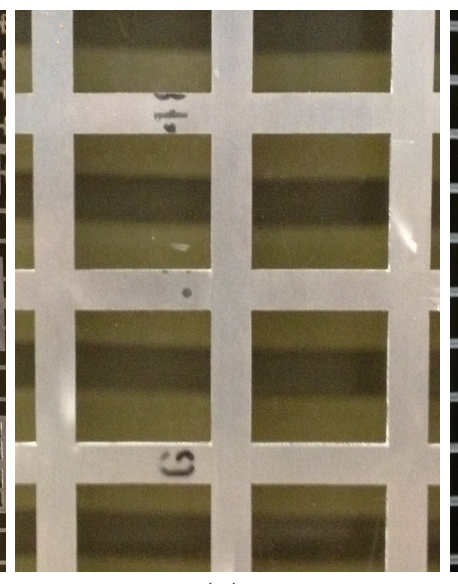

(b)
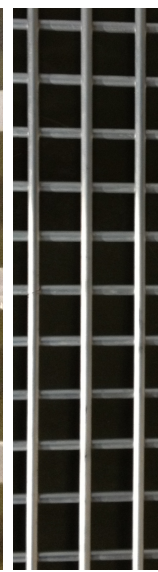

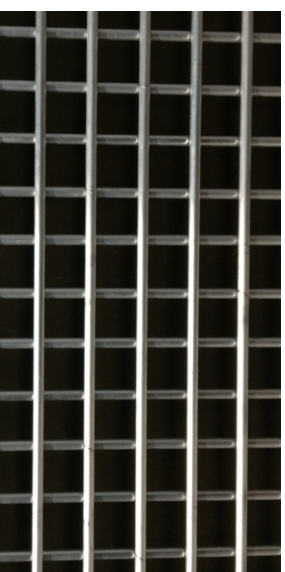

(c)

Figure 1. Photographs of a nominally $260 \mathrm{~mm} \times 340 \mathrm{~mm}$ area of each of the $1200 \mathrm{~mm} \times 800 \mathrm{~mm}$ grids; (a) Fs39, (b) Sq39, (c) $R d 38$.

intensity is less than $0.05 \%$ up to $13 \mathrm{~m} / \mathrm{s}$, monotonically growing to $0.08 \%$ at full speed. The settling chamber consists of a honeycomb and three screens that are specifically manufactured such that there are no seams as to not induce inhomogeneities in the flow. A 9:1 contraction accelerates the flow into the $5 \mathrm{~m}$ long test-section. The test-section itself has an octagonal cross-section that is $0.8 \mathrm{~m}$ high and $1.2 \mathrm{~m}$ wide (see cross-section of figure 2). The corners of the test-section have a nominal length of $0.28 \mathrm{~m}$ with flexible extremities such that they can be adjusted to create a near-zero pressure gradient along the test-section length. The test-section allows for optical access on all eight surfaces. Immediately preceding the test-section is a grid holder where the grids were placed. Downstream of the test-section there are breather vents that equalize the pressure with the room. The test-section is also mechanically isolated from the rest of the wind tunnel by soft connectors used to attach it to the contraction and to the rear diffuser. Finally, before returning to the fan, the flow passes through a heat exchanger that is controlled by an external cooling unit.

For this study, the wind tunnel test-section was modified by adding a slight secondary contraction; this methodology has previously been shown to decrease anisotropy in grid turbulence measurements (Comte-Bellot \& Corrsin 1966; Lavoie et al. 2007). Specifically, the secondary contraction was employed to reduce the variability of the anisotropy in the wakes of the various grids and hence remove it as a driving factor in the analysis of the produced turbulence. The secondary contraction ratio is 1.23:1 and its longitudinal dimensions are given in figure 3. The contraction was composed of $50.8 \mathrm{~mm}$ thick insolation foam attached to the inside walls of the wind tunnel. The profile of the secondary contraction was designed following recommendations by Bell \& Mehta (1988).

The downstream distance relative to the grid was measured by the transit time of the turbulence advection from the grid to a given streamwise position, $x$, (Comte-Bellot \& Corrsin 1966)

$$
t=\int_{0}^{x} \frac{1}{U(s)} \mathrm{d} s,
$$

where $U(x)$ is the local mean velocity at a position $x$, and $s$ is a dummy integration variable. Streamwise position is thus denoted $U_{0} t / M$ rather than the typical $x / M$. For 


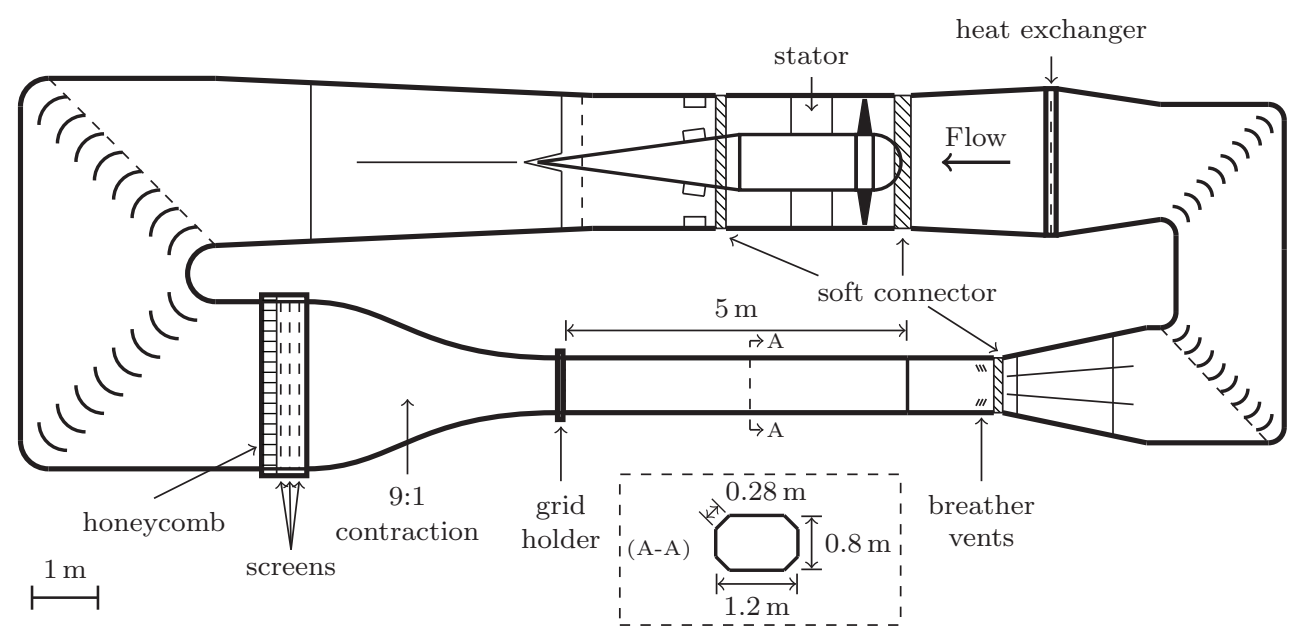

Figure 2. Schematic of the low-speed, recirculating wind tunnel at the University of Toronto Institute for Aerospace Studies.



FIGURE 3. Schematic of the wind tunnel test-section with secondary contraction.

the present study, we also define $U_{c}$ as the measured mean velocity inside the secondary contraction, which is calculated from the mean of $U(x)$ between $1.5 \mathrm{~m} \leqslant x \leqslant 4.0 \mathrm{~m}$.

\subsection{Instrumentation}

Measurements were performed with a Dantec StreamLine constant temperature hotwire anemometer. For streamwise scans, data were acquired with three hot-wire probes simultaneously, a single-wire, a X-wire, and a nano-scale thermal anemometry probe (NSTAP). For transverse scans, data were acquired simultaneously with only the singlewire and $\mathrm{X}$-wire. For both series of tests, the various probes were separated in the $z$ direction by $10 \mathrm{~mm}$. The single-wire and the X-wire were manufactured in-house by mounting $2.5 \mu \mathrm{m}$ diameter tungsten wire to Dantec-style prongs. Sensing lengths were isolated by coating the excess wire with copper. All traditional hot-wire sensing lengths were maintained at $\ell=0.50 \pm 0.05 \mathrm{~mm}$. The separation between the wires of the $\mathrm{X}$-wire was $1.1 \mathrm{~mm}$. The traditional hot-wires were operated at an overheat ratio of 1.6. The NSTAPs were developed at Princeton University by Smits and co-workers (Bailey et al. 2010; Vallikivi et al. 2011). The particular probes used here are described in detail by Vallikivi et al. (2011). An NSTAP is a micro-manufactured single-wire with a $60 \mu \mathrm{m} \times$ $2 \mu \mathrm{m} \times 0.1 \mu \mathrm{m}$ sensing element and a frequency response up to $150 \mathrm{kHz}$, vastly improving the spatial and temporal resolution compared to traditional hot-wires. The NSTAPs were also operated with the StreamLine, but at an overheat ratio of 1.3 as recommended by Vallikivi et al. (2011). The sampling frequency, $f_{s}$, was set to $f_{s}=2 f_{c}+500 \mathrm{~Hz}$, where 


\begin{tabular}{|c|c|c|c|c|c|c|c|c|}
\hline Grid & $\begin{array}{c}U_{c} \\
(\mathrm{~m} / \mathrm{s})\end{array}$ & $\operatorname{Re}_{M}$ & $\begin{array}{c}f_{c} \\
(\mathrm{kHz})\end{array}$ & $\begin{array}{c}t_{s} \\
(\mathrm{~min})\end{array}$ & $\begin{array}{l}\text { Reso } \\
\left(\ell_{u} / \eta\right)\end{array}$ & $\begin{array}{l}\text { ution } \\
\qquad\left(\ell_{v} / \eta\right)\end{array}$ & $\begin{array}{c}\text { Meas. range } \\
\left(U_{0} t / M\right)\end{array}$ & $\begin{array}{l}\text { Max step size } \\
\qquad(\Delta x / M)\end{array}$ \\
\hline$R d 38$ & 17.0 & 28,50 & 30 & 4.0 & {$[0$.} & {$[1$.} & {$[21$.} & 1.41 \\
\hline Fs39 & 5.5 & 28,500 & 10 & 4.1 & {$[0.1,0.3]$} & {$[0.7,2.2]$} & {$[6.9,39.8]$} & 0.45 \\
\hline$S q 39$ & 5.5 & 28,500 & 10 & 4.0 & {$[0.1,0.4]$} & {$[0.8,3.0]$} & {$[6.9,40.5]$} & 0.48 \\
\hline
\end{tabular}

TABLE 2. Experimental setup details for streamwise measurements. All scans were performed along the $(y, z)=(+15,0) \mathrm{mm}$ axis. The square brackets, [.], imply a range of values. The NSTAP and X-wire were used to estimate quantities along these scans.

\begin{tabular}{cccccccc} 
Grid & $\begin{array}{c}U_{c} \\
{[\mathrm{~m} / \mathrm{s}]}\end{array}$ & $R e_{M}$ & $\begin{array}{c}f_{c} \\
{[\mathrm{kHz}]}\end{array}$ & $\begin{array}{c}t_{s} \\
{[\mathrm{~min}]}\end{array}$ & $\begin{array}{c}\text { Resolution } \\
{[\ell / \eta]}\end{array}$ & $\begin{array}{c}\text { Meas. range } \\
{[y / M]}\end{array}$ & $\begin{array}{c}\text { Max step size } \\
{[\Delta y / M]}\end{array}$ \\
\hline$R d 38$ & 20.2 & 32,500 & 30 & 5.0 & {$[1.5,3.0]$} & {$[-8.13,6.56]$} & 1.41 \\
$F s 39$ & 17.4 & 88,500 & 30 & 5.1 & {$[2.1,4.9]$} & {$[-2.50,2.00]$} & 0.35 \\
$S q 39$ & 15.7 & 80,000 & 30 & 5.1 & {$[2.1,4.9]$} & {$[-2.50,2.00]$} & 0.35
\end{tabular}

TABLE 3. Experimental setup details for transverse measurements. Each case was performed at $x=1.25 \mathrm{~m}, 2.00 \mathrm{~m}, 3.25 \mathrm{~m}$, and $4.50 \mathrm{~m}$. This corresponds to $U_{0} t / M=34.2,53.2,84.8$, and 116.5 for $R d 38$, and $U_{0} t / M=10.9,17.0,27.2$, and 37.3 for $F s 39$ and $S q 39$. All scans were performed at $z=0$. A single-wire and X-wire were used to estimate quantities along these scans.

$f_{c}$ is the cut-off frequency of the chosen analog filter. The sample time, $t_{s}$, was chosen such that $\left\langle q^{2}\right\rangle$ was converged to at least $\pm 1 \%$ at every location using the $95 \%$ confidence interval (Benedict \& Gould 1996). Here, $\left\langle q^{2}\right\rangle$ is estimated from $\left\langle q^{2}\right\rangle=\left\langle u^{2}\right\rangle_{\text {NSTAP }}(1+$ $\left.2\left\langle v^{2}\right\rangle_{\mathrm{XW}} /\left\langle u^{2}\right\rangle_{\mathrm{XW}}\right)$. Except when stated otherwise, all results presented for the $u$ velocity component are from the NSTAP. Information on $f_{c}$ and $t_{s}$ for the various tests is provided in tables 2 and 3. The single-wire and NSTAP were calibrated over 10 velocities and fitted with a fourth-order polynomial. The X-wires were calibrated over 10 velocities and 7 angles using the look-up table approach described by Burattini \& Antonia (2005). All calibrations were performed in situ near the end of the test-section where the turbulence intensity was below $2 \%$ for all grids.

To compare $F s 39, S q 39$, and $R d 38$ at a constant $R e_{M}$, streamwise measurements were performed between $0.75 \mathrm{~m}$ and $4.90 \mathrm{~m}$ from the grid along the $(y, z)=(+15,0) \mathrm{mm}$ axis using an automated traverse; $(y, z)=(0,0)$ is the centre of the wind tunnel test-section cross-section, which corresponds to the middle of an opening in $S q 39$ and $R d 38$, and the centre of the background mesh opening for Fs39. This axis was selected because it gave the best approximation of constant $U(x)$ for all grids. Direct comparisons between the turbulence evolution in the wake of the three grids were made only in the far-field where the flow was homogeneous and the choice of axis was thus irrelevant. Near-field comparisons were done on a point-by-point basis using both streamwise and transverse scans and incorporate all statistics at a given point. Measurements were not performed closer to the grid because the pressure had to be allowed to recover downstream of the secondary contraction. We also note that the secondary contraction alters the flow passing through it and as such drawing a direct line back to the point of origin on the grid should be done with caution. Experimental parameters for the streamwise scans are provided in 
table 2. The approximate resolution of the $u$ and $v$ measurements based on the sensing lengths of the wires are also provided in tables 2 and 3 relative to the Kolmogorov microscale $\eta=\nu^{3 / 4} /\langle\epsilon\rangle^{1 / 4}$. The measurement range is given for each streamwise test case in non-dimensional $U_{0} t / M$. Finally, the maximum step size is given as $\Delta x / M$, and indicates the size of the largest step taken between any two streamwise measurements. Some measurements were more finely spaced in regions where increased spatial resolution was desired.

Transverse measurements were acquired downstream of all three grids at $x=1.25 \mathrm{~m}$, $2.00 \mathrm{~m}, 3.25 \mathrm{~m}$, and $4.50 \mathrm{~m}$. The hot-wires were traversed across the test-section using an automated system that spanned $-0.25 \mathrm{~m} \leqslant y \leqslant+0.20 \mathrm{~m}$ along $z=0$. The flow was measured at two mean velocities for each grid; one test at $U_{c}=6 \mathrm{~m} / \mathrm{s}$ and another at the maximum speed that was sustainable in the tunnel. These maximum mean velocities were $U_{c}=20.2,17.4$, and $15.7 \mathrm{~m} / \mathrm{s}$ for $R d 38, F s 39$, and $S q 39$, respectively, and were different because each grid produced a different pressure drop. The two mean velocities were used to ascertain if there was a $R e_{M}$ dependence of the flow homogeneity, but the spanwise scans were not sensitive to $R e_{M}$ at the given stations. As such, we only present the higher $R e_{M}$ case for each grid. Experimental parameters for the transverse measurements are provided in table 3 .

For the streamwise measurements at $R e_{M}=28,500$, the pressure drop, $\Delta P$, across each grid was measured using a 10 torr MKS Baratron transducer. The $\Delta P$ was taken between a static wall port upstream of the grid and the static port on a Pitot static tube located approximately in the centre of the test-section, $2 \mathrm{~m}$ downstream of the grid. This was well downstream of the secondary contraction such that the pressure had recovered. Bernoulli's equation was used to correct the measured pressure drop to the actual pressure drop given the post-grid measurements were performed downstream of the secondary contraction. The normalised pressure drop is thus given by

$$
C_{\Delta P}=\frac{\Delta P}{\frac{1}{2} \rho U_{0}^{2}}=\frac{\Delta P_{\mathrm{m}}}{\frac{1}{2} \rho U_{0}^{2}}-\left(\frac{U_{c}^{2}}{U_{0}^{2}}-1\right),
$$

where $\Delta P_{\mathrm{m}}$ is the measured pressure drop and $\Delta P$ is the true pressure drop across the grid. To produce the same $R e_{M}$ for each grid, the normalised pressure drops were $C_{\Delta P}=0.61,1.21$, and 1.72 for $R d 38, F s 39$, and $S q 39$, respectively. This result contrasts with the DNS of Laizet \& Vassilicos (2015) who found that the pressure drop did not vary significantly with grid design for a given $\sigma$. However, we recognise that our grid bars have different geometries (e.g., round bars compared to square cross-sections). The downstream pressure measurements were performed in a region that can be reasonably identified as the far-field (as discussed in section 3), and the facility was set-up to have an approximately zero-pressure gradient in the test-section. The normalised pressure drop changes by nearly a factor of 3 between $R d 38$ and $S q 39$, and by a factor of 1.4 between $F s 39$ and $S q 39$, both of which are non-negligible changes and well above the uncertainty bounds of the present measurements. As will be shown in section 4, these pressure drop measurements are consistent with the turbulence intensity measured for each grid.

Finally, in order to produce measurements with approximately the same $R e_{\lambda}$ for all grids, a series of scans were performed along the $(y, z)=(+15,0) \mathrm{mm}$ axis at Reynolds numbers spanning $17,500 \leqslant R e_{M} \leqslant 93,000$. The relevant measurements from the $R e_{\lambda}$ matching survey are presented in table 4 . The local Reynolds number used to compare the measurements was that based on the Taylor microscale and assuming isotropy as given in (1.1). This in turn requires computation of the dissipation rate of the turbulent 


\begin{tabular}{cccccc} 
Test & Grid & $U_{0} t / M$ & $y / M$ & $R e_{M}$ & $R e_{\lambda}$ \\
\hline F1a & $F s 39$ & 28.3 & +0.15 & 40,000 & 65 \\
F1b & $F s 39$ & 17.4 & +0.15 & 28,500 & 66 \\
S1 & $S q 39$ & 32.2 & +0.15 & 17,500 & 66 \\
R1 & $R d 38$ & 26.4 & +0.15 & 35,000 & 66 \\
S2 & $S q 39$ & 19.5 & +0.15 & 44,000 & 112 \\
F2a & $F s 39$ & 21.6 & +0.15 & 93,000 & 113 \\
F2b & $F s 39$ & 8.6 & +0.15 & 30,500 & 116 \\
F2c & $F s 39$ & 10.4 & +0.15 & 39,500 & 117 \\
S3 & $S q 39$ & 27.8 & +0.15 & 70,500 & 134 \\
F3a $^{\dagger}$ & $F s 39$ & 10.9 & +1.25 & 88,500 & 134 \\
F3b $^{\dagger}$ & $F s 39$ & 10.9 & +1.00 & 88,500 & 180 \\
F3c $^{\dagger}$ & $F s 39$ & 10.9 & -0.25 & 88,500 & 180 \\
S4 $^{\dagger}$ & $S q 39$ & 17.0 & +0.15 & 84,500 & 180
\end{tabular}

TABLE 4. Details for measurements designed to compare turbulence at the same $R e_{\lambda} \cdot{ }^{\dagger}$ indicates measurements performed with a single-wire. All other measurements were performed with the NSTAP.

kinetic energy for which we use the isotropic definition,

$$
\langle\epsilon\rangle=15 \nu\left\langle\left(\frac{\partial u}{\partial x}\right)^{2}\right\rangle .
$$

The isotropic definitions are used throughout this study because they are those most commonly reported in the literature and are thus easily used for comparison. Furthermore, in previous multi-scale studies it has been demonstrated that estimating the dissipation with different assumptions may marginally influence the magnitude of the estimates but does not change the trends (Hearst \& Lavoie 2014a; Valente \& Vassilicos 2014). Finally, we note that the discussion and conclusions of this work are not influenced by the specific estimate used for the dissipation.

\subsection{Data post-processing and uncertainty analysis}

Post-acquisition, data were low-pass filtered at recursively updated estimates of the Kolmogorov frequency, $f_{K}=U / 2 \pi \eta$, using a fifth-order digital Butterworth filter as described by Mi et al. (2005). This reduces high-frequency noise associated with oversampling the noise floor. The uncertainties associated with finite spatial resolution of the hot-wires were estimated using a Wyngaard (1968) approach. In general, the spatial resolution uncertainty associated with the NSTAP was negligible due to its size being smaller than $\eta$. The spatial resolution uncertainty associated with the estimates of $\left\langle v^{2}\right\rangle$ was less than $0.5 \%$. Gradients were calculated from the time-series with Taylor's frozen flow hypothesis, allowing $x=U t$, and a sixth-order centred-difference scheme that has been shown to provide a good balance of high-frequency noise filtering and resolution for hot-wires (Hearst et al. 2012). Maximum uncertainties on the gradients due to spatial resolution and filtering inherent to the choice of numerical differentiation scheme were $4 \%$ on $\left\langle(\partial u / \partial x)^{2}\right\rangle$ and $10 \%$ on $\left\langle(\partial v / \partial x)^{2}\right\rangle$ (evaluated at the most upstream measurement station), however, these uncertainties decrease with growing $x$ because the smallest scales 
in the flow grow. Estimates of the uncertainties on the presented values are given as error bars or in the captions of the figures throughout this work. In general, the bias uncertainties were estimated with the break-down given by Jørgensen (2002) and the random uncertainties were estimated from the boot-strapping technique described by Benedict \& Gould (1996).

\section{Flow characterisation}

Before in-depth analysis of the turbulence intensity and spectra can be performed, it is relevant to separate the flow into two regions: the non-equilibrium near-field, and the far-field. Here we identify the near-field as the region where the initial generating conditions of the flow still directly influence the evolution of the turbulence. This is identified as the region in the flow that experiences transverse inhomogeneity as well as non-constant evolution of the Reynolds shear stress, $\langle u v\rangle$, and the velocity derivative skewness, $S(\partial u / \partial x)=\left\langle(\partial u / \partial x)^{3}\right\rangle /\left\langle(\partial u / \partial x)^{2}\right\rangle^{3 / 2}$. This is the same technique as that used by Isaza et al. (2014). Further to the above, these metrics have previously been shown to approximately correlate to regions where the dissipation scaling, $C_{\epsilon}$, grows before becoming roughly constant in the far-field (Hearst \& Lavoie 2014a, 2015b).

Transverse scans of the Reynolds shear stress are shown in figure 4 . The wakes of both regular grids have near zero $\langle u v\rangle$ at all transverse measurement stations, while the wake of Fs39 is initially inhomogeneous with transverse oscillations of a period of $M$. As the Fs39 flow evolves, the $\langle u v\rangle$ profile homogenizes between $x=2.00 \mathrm{~m}$ and $3.25 \mathrm{~m}$. In figure 5 it is shown that $\langle u v\rangle \approx 0$ along the $(y / M, z / M)=(0.15,0.0)$ axis for $F s 39$ for $x \gtrsim 2.3 \mathrm{~m}\left(U_{0} t / M \gtrsim 19\right)$. The velocity derivative skewness is also shown in figure 5 , and was found to become approximately constant near $U_{0} t / M=19$ as well. We note that this is quite close to $x / M=20$ established in our previous work for Fs39 without a secondary contraction (Hearst \& Lavoie 2014a,b, 2015b). We thus distinguish the flow ahead of $U_{0} t / M=19$ as the near-field and the flow downstream as the far-field. The transition occurs near $U_{0} t / M=14$ for $S q 39$ (using the same criteria) and the entire measurement range of $R d 38$ is within the far-field. These results for the regular grids corroborate the findings of Isaza et al. (2014). This discrimination is further validated in appendix A with measurements of the transverse transport of turbulent kinetic energy and turbulent production.

In figure $5,\langle u v\rangle$ and $S(\partial u / \partial x)$ are also plotted against $U_{0} t / x^{*}$. Under this normalisation, it would appear that the wake of Fs39 reaches its far-field state sooner in its evolution than that of $S q 39$, an observation made by Isaza et al. (2014) when comparing their regular grid measurements to the Fs39 data from Hearst \& Lavoie $(2014 a)$. However, under $U_{0} t / M$ normalisation and in dimensional units, the wake of $S q 39$ reaches a far-field state (including transverse homogeneity) before that of Fs39; recall $M=L_{0}$ is the same for these two grids. This observation may be more practical given that an application of a turbulence generating technology would likely consider the generated turbulence in either physical units or relative to the mesh length. Moreover, the dissipation appears to scale downstream with $M$ (Hearst \& Lavoie 2014a).

Samples of various turbulent quantities are provided in table 5 to provide understanding of how the various grid wakes evolve. In this work, the integral scale was estimated from,

$$
L_{u}=\frac{1}{\left\langle u^{2}\right\rangle} \int_{0}^{r_{0}}\langle u(x) u(x+r)\rangle \mathrm{d} r,
$$

where $r_{0}$ is the first zero-crossing of the auto-correlation $\langle u(x) u(x+r)\rangle$. From table 5 , 


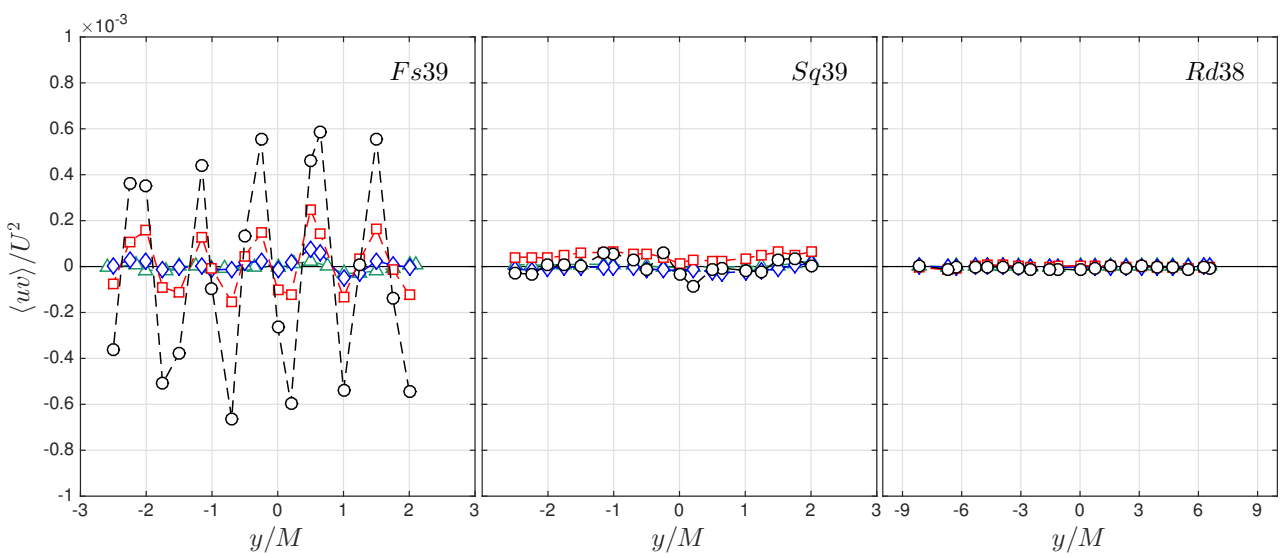

FiguRE 4. Transverse profiles of the normalised Reynolds shear stress for the conditions identified in table $3 ;(\bigcirc) x=1.25 \mathrm{~m},(\square) x=2.00 \mathrm{~m},(\diamond) x=3.25 \mathrm{~m},(\triangle) x=4.50 \mathrm{~m}$. The uncertainty is of the order of the symbol size.
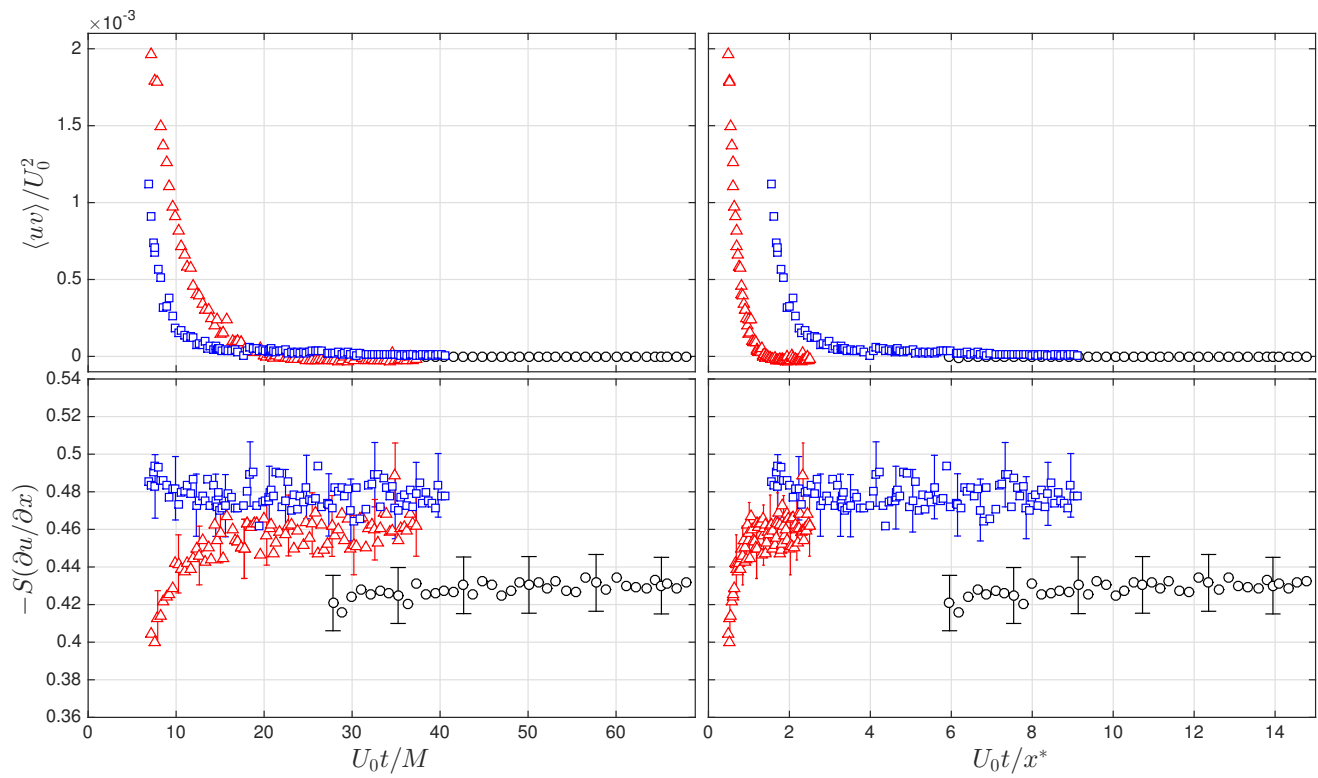

FIGURE 5. Evolution of the Reynolds shear stress and velocity derivative skewness at $R e_{M}=28,500$. $(\triangle) F s 39,(\square) S q 39$, (○) Rd38. The uncertainty of the Reynolds shear stress is of order of the symbol size, and error bars are provided for the velocity derivative skewness.

the global isotropy $\left(u^{\prime} / v^{\prime}\right)$ is collapsed to within $1.10 \pm 0.04$ for $x \geqslant 2 \mathrm{~m}$, which is within the far-field for all flows. This is an improvement on the global isotropy reported without a secondary contraction for both traditional grid turbulence and in our previous measurements behind Fs39 (Hearst \& Lavoie 2014a); typically, $u^{\prime} / v^{\prime} \gtrsim 1.2$. The local isotropy is estimated from the ratio $\left\langle(\partial v / \partial x)^{2}\right\rangle /\left\langle(\partial u / \partial x)^{2}\right\rangle$ which would be 2 in an isotropic flow. The wakes of Fs39 and $R d 38$ both approximate local isotropy to within $5 \%$ for the majority of their evolution and the wake of $S q 39$ is within $10 \%$. We are thus confident that any differences in the far-field turbulence generated by the various grids is not a property of global and local isotropy at a particular position. 


\begin{tabular}{ccccccccccc}
$x$ & $U_{0} t / M$ & $U_{0} t / x^{*}$ & $u^{\prime} / U$ & $R e_{\lambda}$ & $u^{\prime} / v^{\prime}$ & $\frac{\left\langle(\partial v / \partial x)^{2}\right\rangle}{\left\langle(\partial u / \partial x)^{2}\right\rangle}$ & $S\left(\frac{\partial u}{\partial x}\right)$ & $L_{u}$ & $\lambda$ & $\eta$ \\
$(\mathrm{m})$ & & & $(\%)$ & & & & & $(\mathrm{mm})$ & $(\mathrm{mm})$ & $(\mathrm{mm})$ \\
\hline$F s 39$ & & & & & & & & & & \\
1.00 & 8.9 & 0.60 & 5.56 & 104 & 1.19 & 2.16 & -0.42 & 22 & 5.1 & 0.26 \\
2.00 & 17.0 & 1.14 & 2.99 & 66 & 1.14 & 2.06 & -0.45 & 25 & 6.2 & 0.39 \\
3.00 & 25.1 & 1.68 & 2.02 & 53 & 1.09 & 2.09 & -0.46 & 29 & 7.4 & 0.52 \\
4.00 & 33.2 & 2.23 & 1.56 & 48 & 1.07 & 2.12 & -0.46 & 33 & 8.8 & 0.65 \\
4.50 & 37.3 & 2.50 & 1.44 & 48 & 1.11 & 2.04 & -0.46 & 35 & 9.5 & 0.70 \\
\hline \multirow{2}{*}{$S q 39$} & & & & & & & & & & \\
1.00 & 8.9 & 2.00 & 7.49 & 115 & 1.14 & 1.75 & -0.48 & 31 & 4.3 & 0.21 \\
2.00 & 17.0 & 3.83 & 4.12 & 97 & 1.14 & 1.79 & -0.47 & 43 & 6.6 & 0.34 \\
3.00 & 25.1 & 5.65 & 3.04 & 90 & 1.14 & 1.80 & -0.48 & 48 & 8.3 & 0.45 \\
4.00 & 33.2 & 7.48 & 2.48 & 86 & 1.14 & 1.83 & -0.47 & 55 & 9.8 & 0.54 \\
4.50 & 37.3 & 8.39 & 2.25 & 83 & 1.12 & 1.85 & -0.48 & 55 & 10.3 & 0.58 \\
\hline \multirow{2}{*}{$R d 38$} & & & & & & & & & & \\
1.00 & 27.8 & 5.96 & 1.82 & 58 & 1.03 & 2.03 & -0.42 & 12 & 2.9 & 0.20 \\
2.00 & 53.2 & 11.4 & 1.17 & 53 & 1.07 & 2.09 & -0.43 & 16 & 4.1 & 0.29 \\
3.00 & 78.5 & 16.8 & 0.93 & 50 & 1.10 & 2.08 & -0.43 & 18 & 5.0 & 0.36 \\
4.00 & 103.9 & 22.2 & 0.79 & 50 & 1.13 & 2.04 & -0.43 & 21 & 5.8 & 0.42 \\
4.37 & 113.2 & 24.2 & 0.74 & 49 & 1.13 & 2.12 & -0.43 & 26 & 6.2 & 0.44
\end{tabular}

TABLE 5. Flow parameters for all three grids at $R e_{M}=28,500$ for five downstream positions.

\section{Turbulence intensities and Reynolds numbers}

The turbulence intensities and local Reynolds numbers are investigated in figure 6 , which shows $S q 39$ produced the highest turbulence intensities and $R e_{\lambda}$ of the three grids within the measurement range. For the $U_{0} t / M$ range where the $R d 38$ and $F s 39$ data overlap, $R e_{\lambda}$ and the turbulence intensity are higher for $R d 38$. This is to say that for an equivalent streamwise spacing, $U_{0} t / M$, for the square-fractal-element grid and the two regular grids, the regular grids produced higher $R e_{\lambda}$ and turbulence intensity at $R e_{M}=28,500$ within the present measurement range. It was verified that the results are not dependent on the choice of dissipation estimator.

To confirm that the above observations are not simply a consequence of the normalising length scale, the evolution of the turbulence intensity and $R e_{\lambda}$ are also plotted versus $U_{0} t / x^{*}$ and dimensional $x$ in figure 6 . Relative to the wake interaction length scale, the entire measurement range in the wake of Fs39 occurs over less space than the two regular grids, as observed by Isaza et al. (2014). As such, this normalisation corroborates that sufficiently far from the grids Fs39 produces lower turbulence intensities and $R e_{\lambda}$ than equivalent regular grids. In dimensional units, the relative magnitude of the turbulence intensities and $R e_{\lambda}$ between $S q 39$ and $F s 39$ are unchanged, and $R e_{\lambda}$ for $R d 38$ is higher than for Fs39 at the end of the test-section. Thus, sufficiently far from the grids, the two regular grids produce higher $R e_{\lambda}$ than the fractal, in both normalised and dimensional units. 


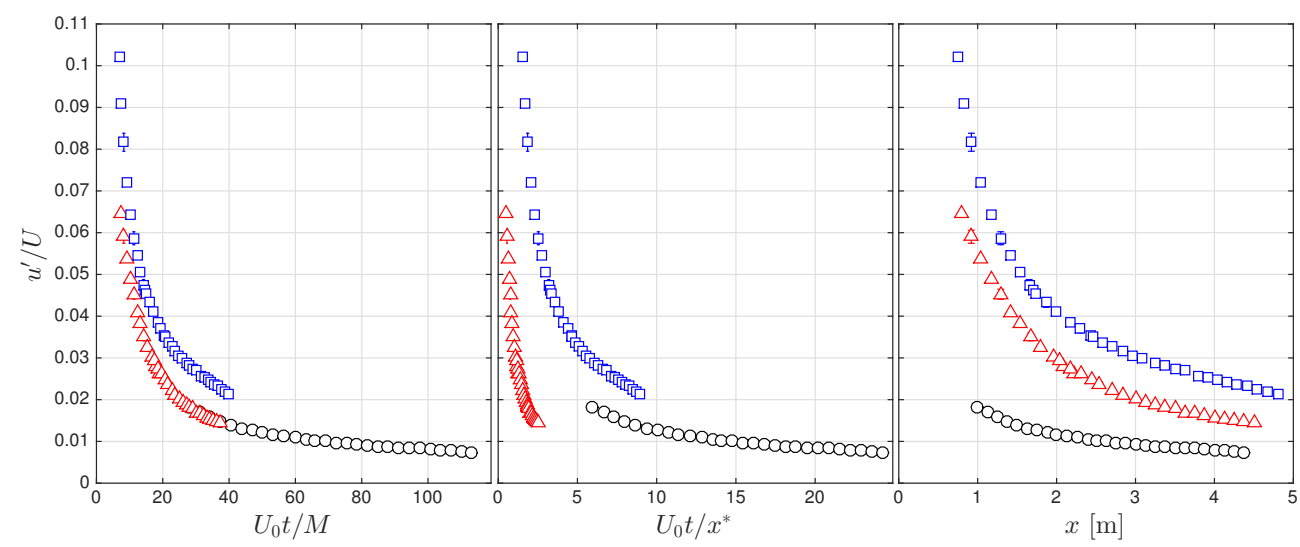

(a)

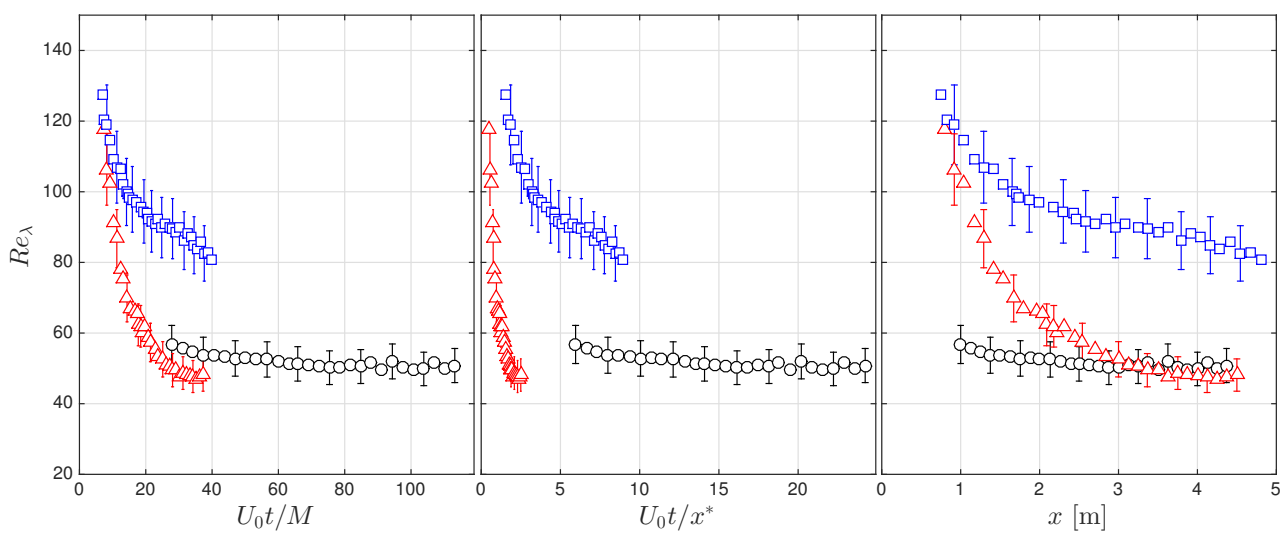

(b)

FiguRE 6. Streamwise evolution of the (a) turbulence intensity and (b) local Reynolds number at $R e_{M}=28,500$. Only every third point is plotted for clarity. $(\triangle) F s 39$, ( $\left.\square\right) S q 39$, (○) $R d 38$.

Ultimately, the far-field of Fs39 is less energetic than that of the regular grids. Insight into this can be found by comparing the decay rates of the turbulent kinetic energy, which, in the far-field, are expected to follow a power-law of the form,

$$
\frac{\left\langle q^{2}\right\rangle}{U^{2}}=A\left(\frac{U_{0} t}{M}-\frac{U_{0} t_{0}}{M}\right)^{n},
$$

where $t_{0}$ is a virtual origin and $A$ is a constant of proportionality. We use the same powerlaw fitting process as described by Hearst \& Lavoie (2014a) to fit power-law unknowns, $A, n$, and $U_{0} t_{0} / M$, over the ranges specified in table 6 where fitting results are also given; results are plotted in figure 7 . The fitting algorithm tested virtual origins within the range $-20 \leqslant U_{0} t_{0} / M \leqslant+20$. Fits were made to both the near- and far-field wakes of Fs39 as distinguished in section 3. The power-laws were also calculated from $\left\langle u^{2}\right\rangle$ and $\left\langle v^{2}\right\rangle$, resulting in $n_{u}$ and $n_{v}$, respectively, which are also provided in table 6 . In general, the estimates of $n, n_{u}$ and $n_{v}$ are consistent to within $5 \%$, except for the nearfield measurements of Fs39 where the disparity is $10 \%$; however, the flow is highly inhomogeneous near Fs39 where one might expect $\left\langle u^{2}\right\rangle$ and $\left\langle v^{2}\right\rangle$ to decay differently. 


\begin{tabular}{c|llcccccc}
$\begin{array}{c}\text { Fit } \\
\#\end{array}$ & Grid & Region & $\begin{array}{c}\text { Fit range } \\
{\left[U_{0} t / M\right]}\end{array}$ & $U_{0} t_{\text {min }} / M$ & $U_{0} t_{0} / M$ & $n$ & $n_{u}$ & $n_{v}$ \\
\hline 1 & $R d 38$ & far-field & {$[21.5,113.2]$} & 27.8 & +3.5 & -1.23 & -1.17 & -1.28 \\
2 & $S q 39$ & far-field & {$[14.2,40.5]$} & 15.6 & +3.0 & -1.32 & -1.32 & -1.30 \\
3 & $F s 39$ & far-field & {$[19.639 .8]$} & 19.6 & +8.0 & -1.25 & -1.25 & -1.27 \\
4 & $F s 39$ & near-field & {$[6.9,19.2]$} & 8.6 & -8.0 & -2.92 & -3.19 & -3.00
\end{tabular}

TABLE 6. Power-law fitting parameters and results.

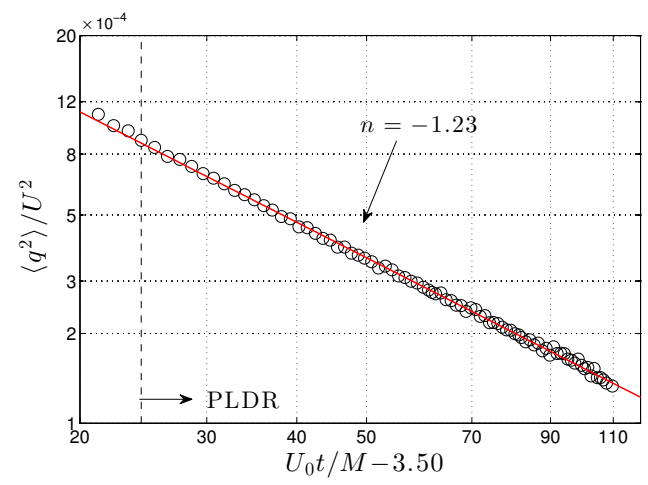

(a)

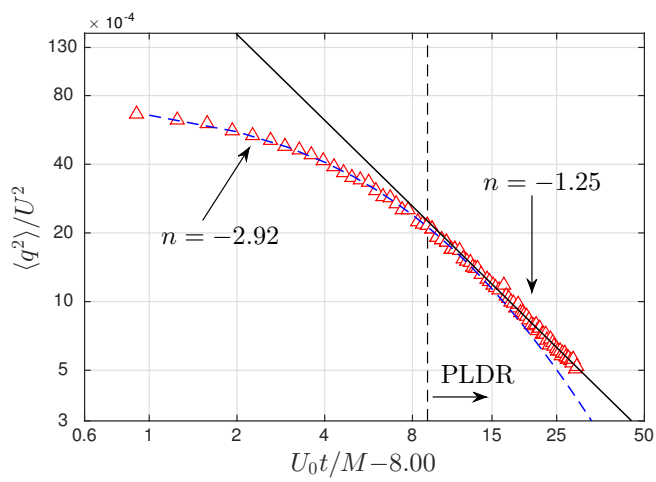

(c)



(b)

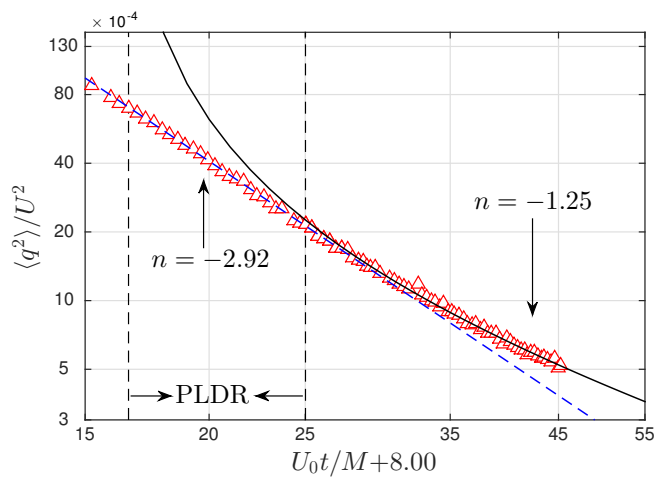

(d)

Figure 7. Decay of turbulent kinetic energy at $R e_{M}=28,500$ with power-law fits for (a) $R d 38$, (b) Sq39, (c) Fs39 far-field, and (d) Fs39 near-field. The power-law decay range (PLDR) is marked by a dashed line. The estimated uncertainty on $\left\langle q^{2}\right\rangle$ is of approximately $\pm 4 \%$ when considering both bias and random uncertainties.

Furthermore, there is no theoretical expectation for a power-law form to the decay in the sheared non-equilibrium near-field, and any such fit would have a spatial dependence. The near-field fit is provided here for a relative comparison to the far-field where the decay rate is lower.

In the far-field wake of all three grids, the power-law exponent is $-1.27 \pm 4 \%$, demonstrating their far-field energy decay is not appreciably different. While this has been implied or suggested by previous studies, this is the first time it has been explicitly 
demonstrated for the same $R e_{M}$ for grids with different geometries but the same blockage (including square fractal). This far-field decay rate is not far removed from the measurements of Sinhuber et al. (2015) who tested a regular grid at a variety of $R_{M}$ using a variable density facility, or Krogstad \& Davidson (2010, 2011) who used both regular and multi-scale cross grids, or the seminal study of Comte-Bellot \& Corrsin (1966). Thormann \& Meneveau (2014) also found values of $-1.16 \geqslant n \geqslant-1.19$ in the wake of their passive square fractal wing grid which is within $6 \%$ of the present measurements.

In the near-field, figure $7(\mathrm{~d})$ clearly shows an extended range (in terms of $U_{0} t / M$ and dimensional units) of rapid energy decay in the wake of $F s 39$ relative to the regular grids. While it is known that this rapid decay range does exist close to the regular grids as well (Krogstad \& Davidson 2012; Isaza et al. 2014), it is drawn out over more $M=L_{0}$ for the square-fractal-element grid. Thus, the produced flow dissipates more of its energy before transitioning to the far-field, resulting in a less energetic far-field. Hence, the far-field wakes of the regular grids have higher turbulence intensities and $R e_{\lambda}$.

These results contrast with the concept promoted by several studies that a fractal produces higher turbulence intensities and Reynolds numbers than an equivalent regular grid (Hurst \& Vassilicos 2007; Valente \& Vassilicos 2011, 2014; Zhou et al. 2014; Laizet \& Vassilicos 2015). However, this is also the first test explicitly comparing an array of fractals to equivalent regular square meshes (relative to $\sigma, M$, and $\tau_{0}$ ), and removing the transverse limitations imposed on the turbulence evolution by the wind tunnel walls, while also allowing for the wakes of multiple regular bars and fractal elements to interact downstream. While this latter fact may not significantly alter the evolution of the square fractal's wake (Weitemeyer et al. 2013), comparing a full mesh with $M$ and $\sigma$ matched to Fs39 is conceivably preferable to comparing a space-filling square fractal to a mesh composed of four bars or with $L_{0} \gg M$. The comparison of Fs39, Sq39, and $R d 38$ highlights that if the high $R e_{\lambda}$ and turbulence intensities that a fractal can produce are desired, one must take careful consideration of the length scales in the grid and the downstream range. Otherwise, it is possible that after a sufficient decay time, a regular grid could provide higher turbulence intensity and $R e_{\lambda}$. These results focus on the far-field of the various grid wakes. For results comparing the near-field of regular and multi-scale grids, we refer the reader to the works by Hurst \& Vassilicos (2007), Krogstad \& Davidson (2012), and Laizet \& Vassilicos (2015).

At this juncture it is necessary to highlight a difference between $F s 39$ and the spacefilling square fractals used in more recent studies. For the present grid, $\tau_{r}=4.5$ (the ratio of thickest to thinnest element in the grid), which is within the range tested by Hurst \& Vassilicos (2007), but is below $\tau_{r}=17$ adopted by more recent studies, e.g., Valente \& Vassilicos $(2011,2014)$, Laizet et al. (2015). The choice of $\tau_{r}$ in the present study was limited by manufacturing constraints balanced with keeping the blockage within acceptable margins. The higher $\tau_{r}$ is desirable because Hurst \& Vassilicos (2007) showed that the flow homogenizes more rapidly in physical space in the wake of a high $\tau_{r}$ space-filling square fractal compared to a similar grid with low $\tau_{r}$. However, even for grids with $\tau_{r}=17$, non-zero production and transverse transport have been measured (Valente \& Vassilicos 2011, 2014), which by definition implies that the flow is not globally homogeneous. Furthermore, in the parametric study performed by Hurst \& Vassilicos (2007), although the peak values of $R e_{\lambda}$ and turbulence intensity grew with $\tau_{r}$, it was shown that after a sufficient streamwise distance $\left(x / M=x / L_{0} \gtrsim 13\right)$, fractals with $2.5 \leqslant \tau_{r} \leqslant 17$ all reached comparable states with respect to turbulence intensity and $R e_{\lambda}$ (see figures 38 and 39 in Hurst \& Vassilicos (2007)). The present measurements do not capture the peak values of the turbulence intensity and $R e_{\lambda}$, since the measurements were restricted - for practical reasons - to the region downstream of the secondary contraction 
$\left(U_{0} t / M \gtrsim 7\right)$. It is therefore not possible to know which grid produces the maximum turbulence intensity and $R e_{\lambda}$ close to the grid. Nonetheless, the data of Hurst \& Vassilicos (2007) suggest that the present results for the far-field of our multi-scale grid would not change appreciably even if a larger value of $\tau_{r}$ was used.

The results presented herein imply that multi-scale grids can produce locally high turbulence intensity and $R e_{\lambda}$ at the cost of a more rapid energy decay beyond the peak of production, while regular grids sustain the turbulence level at farther distances from the grid because of a shorter near-grid rapid decay region. These findings have important implications for a number of engineering and scientific applications requiring the generation of turbulence by grids and demonstrate that the optimal type of grid for a particular application will depend on the goals to be achieved, e.g., peak turbulence intensity, sustained turbulence intensity.

\section{Spectral scaling in the non-equilibrium region}

One of the most puzzling features of the non-equilibrium near-field turbulence is the apparent appearance of a $k^{-5 / 3}$ spectrum in the region where the initial generating conditions' footprint still exists (Isaza et al. 2014; Vassilicos 2015; Laizet et al. 2015). Relative to normalisation by $M$, this occurs fairly close to the grids (Hearst \& Lavoie $2014 a$ ). This result appears paradoxical because $k^{-5 / 3}$ is expected to occur at scales of flows that are free from the initial generating conditions (Frisch 1995; Isaza et al. 2014). The existence of a $k^{-5 / 3}$ inertial region is predicated on there being the same amount of energy entering and leaving the inertial range and thus turbulent production and turbulent transport, for instance, should not influence such scales (although they can be present at larger scales). Laizet et al. (2015) demonstrated that along the centre-line of space-filling square fractals and large four bar regular grids the frequency spectrum exhibits $f^{-5 / 3}$ in the production region before the peak of the turbulence intensity. Farther downstream, where Taylor's hypothesis can be applied, this manifests as a $k^{-5 / 3}$ spectral region that eventually decays away from $-5 / 3$ with streamwise distance from the grid (Laizet et al. 2015), presumably due to decaying $R e_{\lambda}$ (Mydlarski \& Warhaft 1996; Isaza et al. 2014). However, this has not been explored off-axis. Off-axis measurements can contribute valuable insight into the conditions required to steepen the scaling range spectrum toward $k^{-5 / 3}$ or perhaps passed it. Recall that sheared regions with $\langle u v\rangle \neq 0$ in both turbulent boundary layers (Saddoughi \& Veeravalli 1994) and turbulent jets (Mi \& Antonia 2001) also produce near $k^{-5 / 3}$ spectra. In fact, Mi \& Antonia (2001) showed that in highly sheared regions of the flow where there was also significant largescale intermittency it was possible for the spectral slope to exceed $k^{-5 / 3}$, and that shear and large-scale intermittency were more important factors in determining the spectral topology than $R e_{\lambda}$. We thus seek to clarify if the apparent appearance of $k^{-5 / 3}$ in the near wake of grids is ubiquitous across the non-equilibrium flow field, and what properties are present when such a spectrum is observed. This is addressed by comparing the spectra at constant $R e_{\lambda}$ in the non-equilibrium region and far-field of both multi-scale and regular grids.

First, the spectra $\left(\left\langle u^{2}\right\rangle=\int_{0}^{\infty} F_{11}(k) \mathrm{d} k\right)$ are compared in the far-field wakes of all three grids. Figure 8 shows the compensated spectra for each grid at $R e_{\lambda} \approx 66$. To generate the same $R e_{\lambda}$, the downstream position and $R e_{M}$ had to be varied between test cases, as detailed in table 4 . Despite the relatively low $R e_{\lambda}$, the spectra appear to be approximately independent of $R e_{M}$ and grid geometry as the four curves from the three grids are approximately collapsed at all wavenumbers. The significance of this finding is two-fold. First, the spectrum from the multi-scale grid is indistinguishable from that 


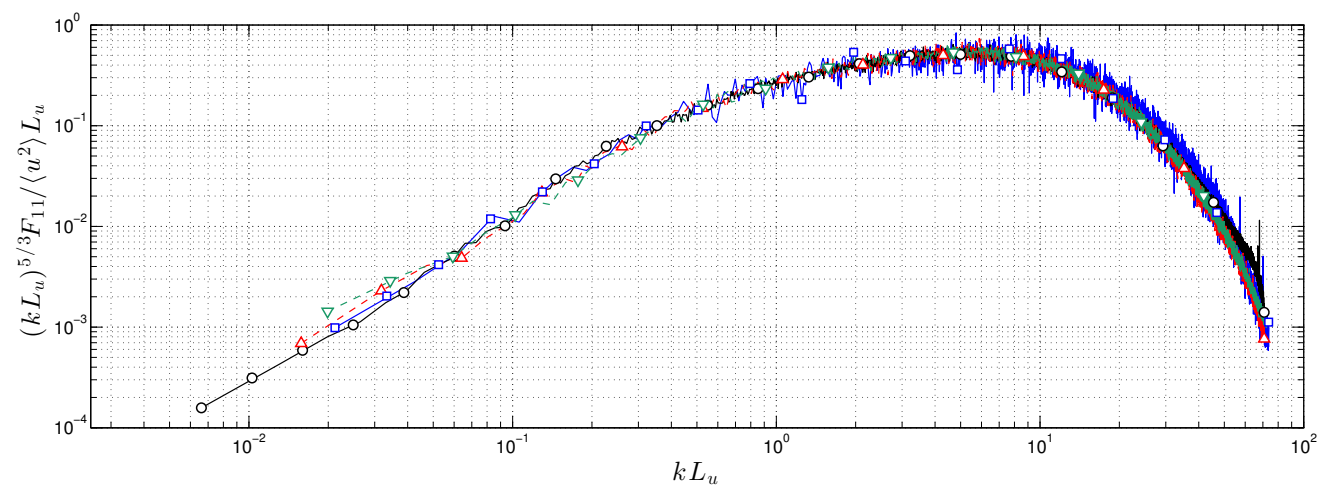

FiguRE 8 . Compensated spectra at $R e_{\lambda} \approx 66$ in the far-field of each grid along $y / M=+0.15$. $(\triangle) \mathrm{F} 1 \mathrm{a}, F s 39, U_{0} t / M=28.3, R e_{M}=40,000, R e_{\lambda}=65 ;(\nabla) \mathrm{F} 1 \mathrm{~b}, F s 39, U_{0} t / M=17.4$, $R e_{M}=28,500, R e_{\lambda}=66$; ( $\left.\square\right) \mathrm{S} 1, S q 39, U_{0} t / M=32.2, R e_{M}=17,500, R e_{\lambda}=66$; (○) R1, $R d 38, U_{0} t / M=26.4, R_{M}=35,000, \operatorname{Re}_{\lambda}=66$.

of the regular grids in the far-field. No previous study has explicitly observed this nor suggested it might be the case, instead they have focussed on the existence of an apparent $k^{-5 / 3}$ spectrum in the non-equilibrium region without performing detailed investigations farther downstream at the same $R e_{\lambda}$. Second, the fact that the spectra from all three grids are remarkably similar demonstrates that the spectral distribution of the energy has predominately forgotten its generating conditions, at least at intermediate and small scales, even at this very low $R e_{\lambda}$. A similar result was observed by Hearst \& Lavoie (2015a) using an active grid for $R e_{\lambda}$ up to 485. They used various grid operational settings to produce the same $R e_{\lambda}$ and found the scaling and dissipative ranges of the spectra approximately collapsed despite their very different initial generating conditions.

The comparison of all three grids at one $R e_{\lambda}$ is limited by the relatively low Reynolds numbers produced by $R d 38$. From this point forward, we compare only $S q 39$ and $F s 39$ so that higher $R e_{\lambda}$ are achievable. In figure 9 , the compensated spectra measured at $R e_{\lambda} \approx 115$ from both the near- and far-fields of Fs39 are compared to the far-field of $S q 39$. Although the $R e_{\lambda}$ is matched, the scaling range transitions to the dissipation range at a higher wavenumber for the far-field turbulence compared to the non-equilibrium near-field turbulence. The non-equilibrium turbulence produces a scaling range that is closer to, but is still different from, $k^{-5 / 3}$. This result is verified for different $R e_{M}$ and in the far-field of both Fs39 and $S q 39$. Hence, degradation away from or an approach toward a scaling range slope of $k^{-5 / 3}$ cannot be solely related to variations in $R e_{\lambda}$ as is oft reported (Mydlarski \& Warhaft 1996; Isaza et al. 2014), but must be related to local properties that are evolving (Laizet et al. 2015).

It is significant to remind the reader that there is no theoretical expectation for a $k^{-5 / 3}$ inertial range in a highly sheared flow with production and transverse transport, such as that in the near wake of grids (Kolmogorov 1941; Frisch 1995; Isaza et al. 2014). Rigorously, it may be argued that for "an arbitrary turbulent flow with a sufficiently large Reynolds number the hypothesis of local isotropy is realised with good approximation in sufficiently small domains ... not lying near the boundary of the flow or its other singularities" (Kolmogorov 1941, p. 10). However, if these conditions were achieved here, then the spectra should not show a dependence on their measurement in the nonequilibrium region or far-field. Thus, the range of $R e_{\lambda}$ here is insufficient for manifestation of a $k^{-5 / 3}$ range in the non-equilibrium region based on Kolmogorov's framework. 




FIGURE 9. Comparison of compensated spectra at $R e_{\lambda} \approx 115$ from both the near- and far-field of $S q 39$ and $F s 39$ along $y / M=+0.15$. ( $\square)$ S2, far-field, $S q 39, U_{0} t / M=19.5, R e_{M}=44,000$, $R e_{\lambda}=112 ;(\triangle)$ F2a, far-field, $F s 39, U_{0} t / M=21.6, R e_{M}=93,000, R e_{\lambda}=113 ;(\nabla) \mathrm{F} 2 \mathrm{~b}$, near-field, $F s 39, U_{0} t / M=8.6, R e_{M}=30,500, R e_{\lambda}=116 ;(\triangleright)$ F2c, near-field, Fs 39 , $U_{0} t / M=10.4, R e_{M}=39,500, R e_{\lambda}=117$. Only F2a and F2b are plotted in the inset for clarity.

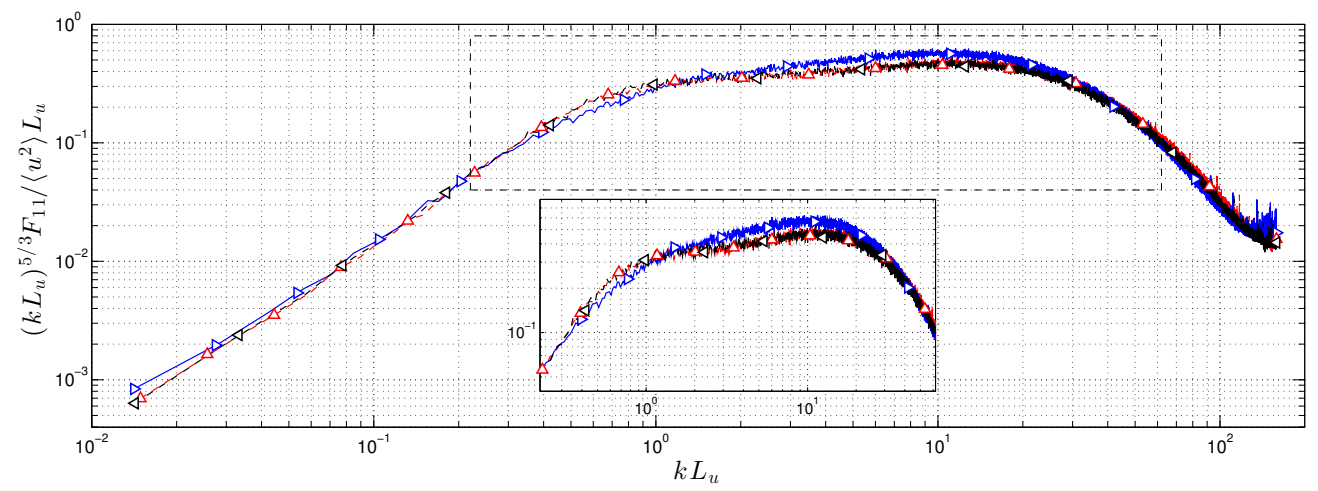

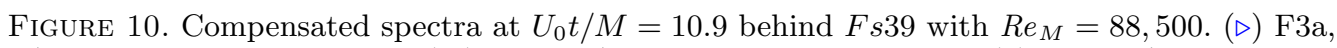
$y / M=+1.25, R e_{\lambda}=134 ;(\triangle) \mathrm{F} 3 \mathrm{~b}, y / M=+1.00, R e_{\lambda}=180 ;(\triangleleft) \mathrm{F} 3 \mathrm{c}, y / M=-0.25$, $R e_{\lambda}=180$. These spectra are estimated using a single-wire.

Nonetheless, the slope of the scaling range is closest to $-5 / 3$ in the non-equilibrium region, and we thus seek to understand what causes the spectrum to increase in slope.

To determine how the spectra change in the non-equilibrium region, three spectra from the transverse scan of $F s 39$ at $U_{0} t / M=10.9$ with $R e_{M}=88,500$ are shown in figure 10 . The two curves with scaling ranges nearer $k^{-5 / 3}$ correspond to peaks of opposite magnitude in the Reynolds shear stress $\left(\left|\langle u v\rangle / U^{2}\right| \approx 6 \times 10^{-4}\right)$ as shown in figure 4, which results in production on the order of $30 \%$ of the dissipation (Appendix A). In contrast, the spectrum that deviates the most from $k^{-5 / 3}$ occurs at a transverse location that experiences negligible Reynolds shear stress and thus no production. This suggests that the spectra with a steeper slope are linked to locations where $\langle u v\rangle \neq 0$. Note that in general locations where $\langle u v\rangle \neq 0$ correspond to locations where there is production (compare figures 4 and 16).

In figure 10, all the spectra do not have the same $R e_{\lambda}$. The two spectra that lie closest to $k^{-5 / 3}$ have $R e_{\lambda}=180$, while the other is for a case where $R e_{\lambda}=134$. To address this issue, figure 11(a) compares one of the curves with $\langle u v\rangle \neq 0$ from figure 10 to a spectrum from the far-field of $S q 39$ at $R e_{M}=84,500$ where $\langle u v\rangle=0$ but where $R e_{\lambda}=180$. Here, 


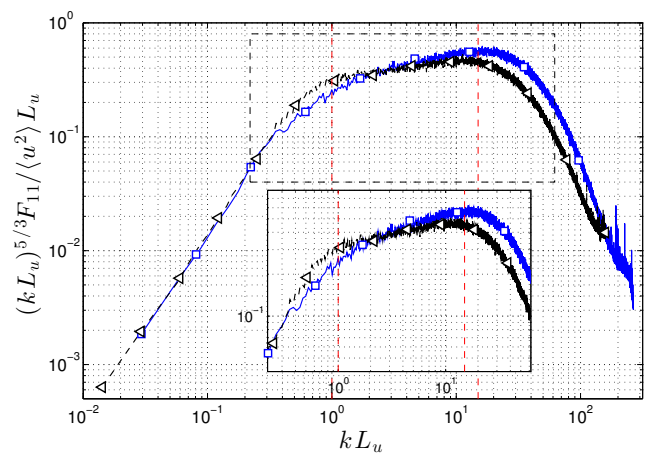

(a)

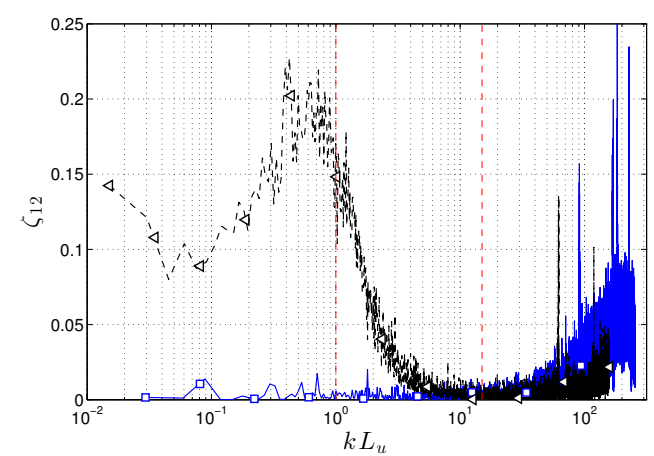

(b)

Figure 11. Comparison of (a) compensated spectra and (b) spectral coherence as measured in the non-equilibrium near-field and the far-field at $R e_{\lambda}=180$. ( $\left.\triangleleft\right)$ F3c, near-field, Fs39, $U_{0} t / M=10.9, y / M=-0.25, R e_{M}=88,500$; $(\square)$ S4, far-field, $S q 39, U_{0} t / M=17.0$, $y / M=+0.15, R e_{M}=84,500$. Vertical lines roughly identify the scaling range. $F_{11}$ is measured using a single-wire and $\zeta_{12}$ is measured with a X-wire.

at a higher $R e_{\lambda}$ than figure 9, we again demonstrate that we can have two different spectral shapes for the same $R e_{\lambda}$ if the two flows have different contributions from the Reynolds shear stress.

While it would appear that $\langle u v\rangle$ has an influence on the spectral shape in the scaling range, this should only be possible if $\langle u v\rangle$ penetrates to scales that overlap with the scaling range. A true $k^{-5 / 3}$ inertial range as described by Kolmogorov (1941) can only exist under very specific circumstances, one of which is that there is a sufficient separation of scales such that there is no production at scales associated with the inertial range. The region with a steeper slope that is nearer $k^{-5 / 3}$ in figure 11 is highlighted with vertical lines at $k_{a} L_{u}=1$ and $k_{b} L_{u}=15$. As we know that there is Reynolds shear stress present in instances where the scaling range slope is steeper for a given $R e_{\lambda}$, and $\langle u v\rangle$ is in the production term, we can gain insight on the scales influenced by production from the cross-spectral coherence,

$$
\zeta_{12}=\frac{\left|F_{12}\right|^{2}}{F_{11} F_{22}},
$$

where $\left\langle v^{2}\right\rangle=\int_{0}^{\infty} F_{22} \mathrm{~d} k$, and $\langle u v\rangle=\int_{0}^{\infty} \Re\left\{F_{12}\right\} \mathrm{d} k$. The coherence in the nonequilibrium region of $F s 39$, shown in figure $11(\mathrm{~b})$, has a peak at $20 \%$ near $k L_{u}=0.7$ that remains non-zero for the large scales and decreases for increasing wavenumbers. For $k_{a} \leqslant k \leqslant k_{b}, \zeta_{12}$ drops from $15 \%$ at $k L_{u}=1$ to approximately zero at $k L_{u}=8$, which contrasts with $\zeta_{12} \approx 0$ for all $k$ in the wake of $S q 39$. Quantitatively, the contributions to the normalised Reynolds shear stress from the range $k_{a} \leqslant k \leqslant k_{b}$ (i.e., $\left.\left(1 / u^{\prime} v^{\prime}\right) \int_{k_{a}}^{k_{b}} \Re\left\{F_{12}\right\} \mathrm{d} k\right)$ is $9.6 \%$ for $F s 39$, compared to approximately zero for $S q 39$. The evolution of $\zeta_{12}$ in $U_{0} t / M$ is shown for $F s 39$ at $R e_{M}=28,500$ in figure 12, further identifying that as one moves out of the non-equilibrium region, $\zeta_{12} \rightarrow 0$ for all $k$. This is consistent with the evolution of $\langle u v\rangle$ for the same flow reported in figure 5 , and demonstrates both that the correlation between $u$ and $v$ diminishes with position away from the grid and that the scales influenced by this correlation include the scaling range until the far-field is reached.

The inference of the above is that production at scales that overlap with a steeper scaling range results in a spectrum that appears nearer to $k^{-5 / 3}$ compared to a position in the flow at the same $R e_{\lambda}$ without production. However, the kinematics in spectral space 


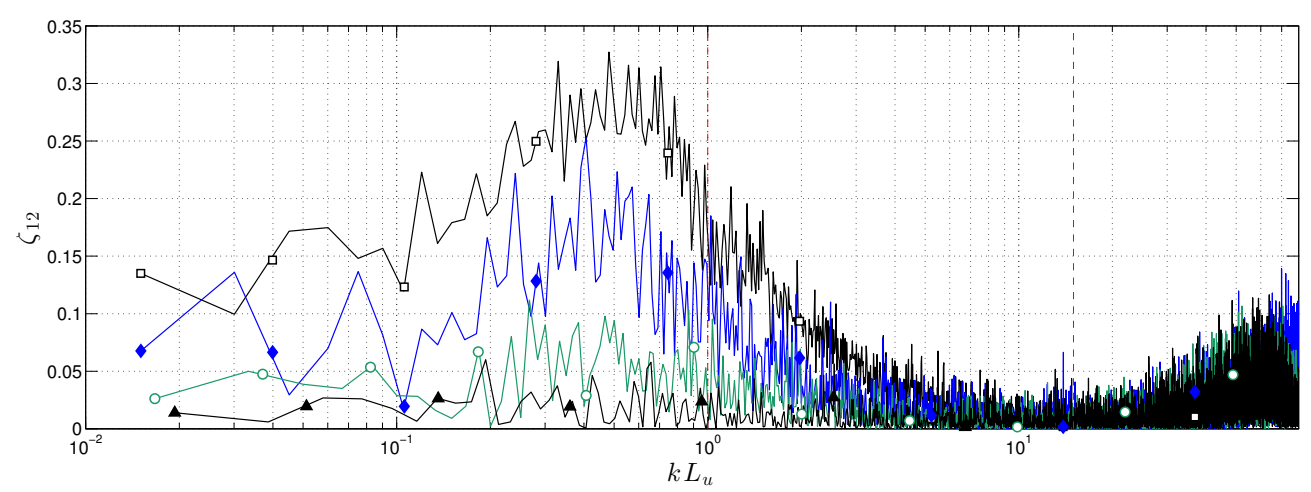

FiguRE 12. Spectral coherence at several locations downstream of $F s 39$ at $R e_{M}=28,500$. ( $\square) U_{0} t / M=6.9,(\diamond) U_{0} t / M=9.9,(\bigcirc) U_{0} t / M=13.3,(\boldsymbol{\Delta}) U_{0} t / M=16.7$.

are non-linear and thus coherence at scales that overlap with the 'inertial range' does not necessitate a change to the energy balance and $F_{11}$ at those scales. The structure function analog of $F_{11} \sim k^{-5 / 3}$ is $\left\langle(\delta u)^{2}\right\rangle \sim r^{2 / 3}$, and the latter relationship is that actually reported by Kolmogorov $(1941) ;\left\langle(\delta u)^{2}\right\rangle=\left\langle(u(x+r)-u(x))^{2}\right\rangle$ is the secondorder structure function. With respect to the equations for the structure functions, the various terms of the energy budget sum linearly, and hence the existence of Reynolds shear stress on a scale-by-scale basis, as indicated by $\langle(\delta u)(\delta v)\rangle$ can be assessed.

Figure 13 shows both the compensated $\left\langle(\delta u)^{2}\right\rangle$ and native $\langle(\delta u)(\delta v)\rangle$ for the same cases as shown in figure 11. The approximate scaling range is highlighted by the dashed vertical lines. The dashed and dashed-dotted lines represent the $r>0$ and $r<0$ sides of $\langle(\delta u)(\delta v)\rangle$, respectively. It is clear that there is no significant content in $\langle(\delta u)(\delta v)\rangle$ at any scale in the wake of $S q 39$ for a position where $\langle u v\rangle \approx 0$, which contrasts starkly with changing $\langle(\delta u)(\delta v)\rangle$ in the range that overlaps with the scales associated with the steeper scaling range for the same $R e_{\lambda}$ for $F s 39$. Thus, figures 11-13 clearly identify that not only is $\langle u v\rangle \neq 0$ but that it also penetrates to scales that overlap with the perceived 'inertial range'.

In order to confirm that it is the presence of local $\langle u v\rangle$ that manipulates the shape of the spectrum, we compare a near-field non-equilibrium spectrum measured at a position with $\langle u v\rangle \approx 0$ to a far-field spectrum at the same $R e_{\lambda}$ and also with $\langle u v\rangle \approx 0$ in figure 14. The figure once again confirms that for a given $R e_{\lambda}$ the compensated spectra are approximately collapsed. This result is quite remarkable given it is observed between a near-field non-equilibrium spectrum and a far-field spectrum from different grids. It also strongly suggests that the steepening of the scaling range slope and the early onset of a near $k^{-5 / 3}$ spectrum observed in previous studies is related to local properties of the flow rather than being a general consequence of non-equilibrium phenomenology.

The steepening of the scaling range slope in the non-equilibrium region has been primarily attributed to the existence of shear forces that exist at a range of scales that overlap with the scaling range. In the non-equilibrium region the velocity derivative statistics also have a spatial dependence, e.g., figure 5 and Hearst \& Lavoie $(2015 b)$. This could suggest internal intermittency plays a role in the present observations (Kolmogorov 1962; Mi \& Antonia 2001). However, we have focussed on the Reynolds shear stress because it is demonstrable that it influences scales where a steepened scaling range slope was observed. Furthermore, previous studies have demonstrated internal intermittency causes deviations away from $k^{-5 / 3}$, i.e., a flattening of the scaling range slope rather than a steepening of it. Withal, in flows such as these where the large scale shear penetrates 


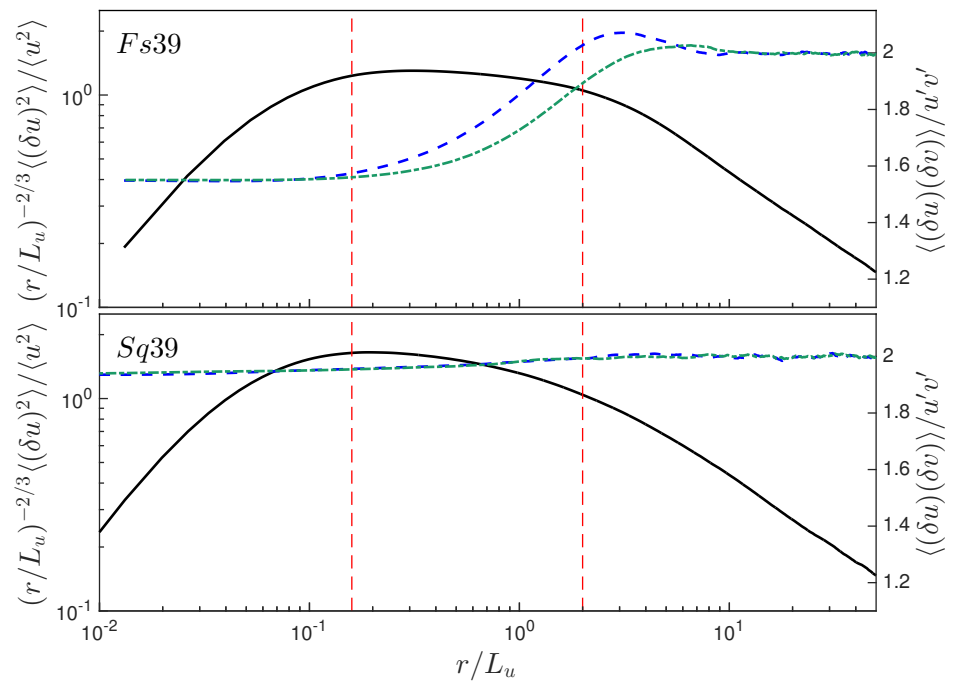

Figure 13. Second-order structure functions. (Top) F3c, near-field, Fs39, $U_{0} t / M=10.9$, $y / M=-0.25, R e_{M}=88,500$; (bottom) S4, far-field, $S q 39, U_{0} t / M=17.0, y / M=+0.15$, $\operatorname{Re}_{M}=84,500$. (-) $\left\langle(\delta u)^{2}\right\rangle,(--)\langle(\delta u)(\delta v)\rangle$ for $r>0,(-\cdot-)\langle(\delta u)(\delta v)\rangle$ for $r<0$. Vertical lines roughly identify the scaling range. These results were measured by a X-wire.

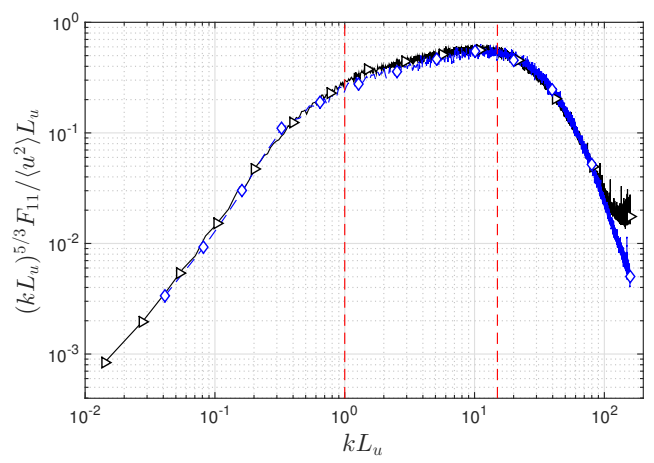

(a)

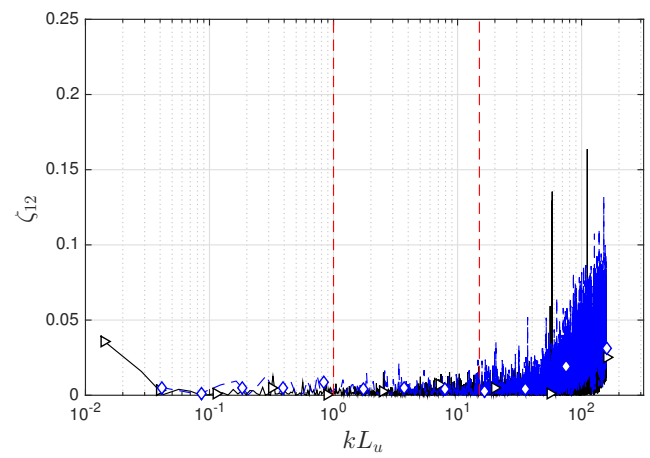

(b)

Figure 14. Comparison of (a) compensated spectra and (b) spectral coherence as measured in the non-equilibrium near-field and the far-field at $R e_{\lambda}=134$ for cases where $\langle u v\rangle \approx 0$. $(\triangleright)$ F3a, near-field, $F s 39, U_{0} t / M=10.9, y / M=+1.25, R_{M}=88,500 ;(\diamond)$ S3, far-field, $S q 39$, $U_{0} t / M=27.8, y / M=+0.15, R e_{M}=70,500$. Vertical lines roughly identify the scaling range. $F_{11}$ is measured using a single-wire and $\zeta_{12}$ is measured with a X-wire.

to the intermediate scales in the spectra and structure functions, $k^{-5 / 3}$ may not in fact be the asymptote. For instance, Mi \& Antonia (2001) showed scaling ranges with slopes that exceed $-5 / 3$ in the sheared region of a turbulent jet.

There is some evidence in other measurements to support the correlation observed here. Saddoughi \& Veeravalli (1994) investigated the assumption of local isotropy in a large turbulent boundary layer. This flow included both mean shear and $\langle u v\rangle$. For cases where they observed a near $k^{-5 / 3}$ inertial range, they also noticed that $F_{12}$ did not become approximately zero for at least a decade after the start of the $k^{-5 / 3}$ region. Thus, their flow also experienced a steep scaling range slope at scales influenced by $\langle u v\rangle$. In the intermittent and highly sheared periphery of a turbulent jet, Mi \& Antonia (2001) 
observed a steepening of the scaling range slope. In their case, they measured slopes that exceeded $k^{-5 / 3}$, which is a reminder that in such flows where the small scales are not independent of the large scales there is no expectation of $k^{-5 / 3}$ nor is it necessarily an asymptote. Their results cannot be directly related to the presence of $\langle u v\rangle$ because they did not report measurements of this parameter, however, it is known to be non-zero in the region of their measurements. More recently, Vassilicos and co-workers have shown in various studies that a $k^{-5 / 3}$ range that is up to two decades long manifests in the nonequilibrium region of the wake of multi-scale grids (Hurst \& Vassilicos 2007; Seoud \& Vassilicos 2007; Mazellier \& Vassilicos 2010; Valente \& Vassilicos 2011; Laizet et al. 2015). It has been demonstrated that the non-equilibrium region of these flows experiences nonzero production (Hurst \& Vassilicos 2007; Nagata et al. 2013; Hearst \& Lavoie 2014b), which implies $\langle u v\rangle \neq 0$. Furthermore, comparing the coherence in figure $4(\mathrm{c})$ to the compensated spectra in figure 15(c) of Valente \& Vassilicos (2011) shows that there is some overlap of the non-zero coherence with the start of the scaling range (which is reminiscent of the results of Saddoughi \& Veeravalli (1994)), and figure 14 of the same work shows that for similar $R e_{\lambda}$, the spectrum is nearer to $k^{-5 / 3}$ for the multi-scale generated non-equilibrium turbulence. Laizet et al. (2015) focussed on the appearance of a $-5 / 3$ region in the spectra and traced it to the production region immediately behind the grid where $\langle u v\rangle \neq 0$ and $k^{-5 / 3}$ is not expected. They clearly state that the appearance of a $-5 / 3$ region is not related to the Kolmogorov framework. Finally, for regular grids, Isaza et al. (2014) have also observed steeper slopes in the scaling range for measurements in the non-equilibrium region near their grid where they report $\langle u v\rangle / u^{\prime} v^{\prime} \neq 0$. Thus, in addition to the results reported here, there is significant evidence in the literature suggesting there is a correlation between regions that experience $\langle u v\rangle$ and steepening of the scaling range spectral slope.

\section{Conclusions}

Two regular grids were compared to a square-fractal-element grid in order to investigate key phenomenological differences between the turbulence produced by a multi-scale fractal-based geometry and regular grids. The square-fractal-element grid, Fs39, was composed of an array of square fractal elements mounted to a background mesh. A square bar regular grid, Sq39, was designed with matched $M=L_{0}$ and $\sigma$ to Fs39. A round-rod regular grid, $R d 38$, was designed with $\tau_{0}$ and $\sigma$ approximately matched to Fs39. A secondary contraction was employed downstream of the grids in order to decrease the anisotropy in the flow and facilitate distinction of the turbulence produced by each grid based on parameters other than anisotropy levels.

When compared at constant $R e_{M}$, it was demonstrated that the regular grids produced higher turbulence intensities and $R e_{\lambda}$ in the far-field than the multi-scale geometry used here. In particular, $S q 39$ produced higher turbulence intensities and $R e_{\lambda}$ for the entire investigation range. It is possible the peak turbulence intensity in the wake of $F s 39$ exceeded that of $S q 39$, but the present measurements show that if this were the case, it is not sustained beyond $U_{0} t / M>7$. Furthermore, both regular grids homogenized more rapidly than the fractal. These results may suggest that the regular grids act as more efficient mixers than the present multi-scale geometry, particularly given that one of the regular grids also had a lower pressure drop than the multi-scale geometry. Future studies investigating the evolution of passive scalars in the wakes of comparable regular and multi-scale grids may provide further insight on this topic. Regardless, the primary influence of the fractal geometry appears to be to extend the non-equilibrium near-field region in the wake of the grid. This region experiences rapid decay of the turbulent 
kinetic energy, and by extending it the fractal essentially creates a less energetic far-field relative to the regular grids. This is the first reporting of a flow where a regular grid with similar properties to a square-fractal-based geometry clearly produces higher turbulence intensities and $R e_{\lambda}$ in the far-field of its evolution for the same global $R e_{M}$. This result is particularly significant for engineers who wish to use turbulence generators in practical applications, as it demonstrates that the 'beneficial' properties of the fractal only persist for a finite period of the decay, and if sustained homogeneous turbulence levels and $R e_{\lambda}$ are required, a regular grid may be better suited for the application.

The steepening of the velocity spectrum scaling range slope in the non-equilibrium region was shown to be a phenomenon dependent, at least in part, on the local $\langle u v\rangle$. When a near-field, non-equilibrium spectrum with $\langle u v\rangle \neq 0$ was compared to a far-field spectrum at the same $R e_{\lambda}$, the non-equilibrium spectrum was steeper. For these nonequilibrium spectra with steeper scaling range slopes for a given $R e_{\lambda}$ and $\langle u v\rangle \neq 0$, it was observed that the Reynolds shear stress penetrated to scales that overlapped with the scaling range. In contrast, when a spectrum from the non-equilibrium region with $\langle u v\rangle \approx 0$ was compared to a far-field spectrum also with $\langle u v\rangle \approx 0$ at the same $R e_{\lambda}$, they were not appreciably different, irrespective of which grid produced the flow. Some previous studies have associated this steepened scaling range with a $k^{-5 / 3}$ region, although they are always careful to note that this is not a Kolmogorov-based inertial range, e.g., Mazellier \& Vassilicos (2010), Laizet et al. (2015). The present results show that the near $k^{-5 / 3}$ spectrum in the non-equilibrium region is not a universal property of this region and is rather dependent on local properties, particularly $\langle u v\rangle$.

The authors acknowledge the financial support of the Natural Sciences and Engineering Research Council of Canada (NSERC). They would also like to thank Prof. A. J. Smits of Princeton University for providing the NSTAPs, and Prof. A. Pollard of Queen's University for providing the Dantec StreamLine system. RJH would like to thank Dr. M. Vallikivi for the time she spent training him on the set-up, use, and repair of NSTAPs, and Dr. H. Clark for her valuable insights on the analysis.

\section{Appendix A. Turbulent production and transverse transport}

This appendix presents additional statistics from the transverse scans behind all three grids in order to help paint a full picture of the evolution of the turbulence. Figure 15 shows transverse profiles of the mean velocity normalised by the mean of $U(y)$ across a transverse scan, denoted $U_{y}$, for each grid. From the first measurement position $(x=$ $1.25 \mathrm{~m})$ behind $S q 39$ and $R d 38\left(U_{0} t / M=10.9\right.$ and $U_{0} t / M=34.2$, in non-dimensional units, respectively) the flow is homogeneous to within $\pm 1 \%$. The homogeneity of $U$ in the wake of Fs39 contrasts with the regular grid results. At $x=1.25 \mathrm{~m}\left(U_{0} t / M=\right.$ 10.9 ) the mean velocity varies in the range $\pm 4 \%$, however, it homogenizes to $\pm 2 \%$ by $x=2.00 \mathrm{~m}\left(U_{0} t / M=17.0\right)$; this is similar to the results presented by Hearst \& Lavoie $(2014 a, b)$ without a secondary contraction. For $U_{0} t / M \geqslant 17.0$, the flow is approximately homogeneous with respect to $U$.

Production and transverse transport of turbulent kinetic energy have been identified as key differentiating factors between non-equilibrium turbulence and theoretical equilibrium homogeneous, isotropic turbulence (Valente \& Vassilicos 2011, 2014; Nagata et al. 2013; Hearst \& Lavoie 2014b). Assessment of these parameters is done in the context of 


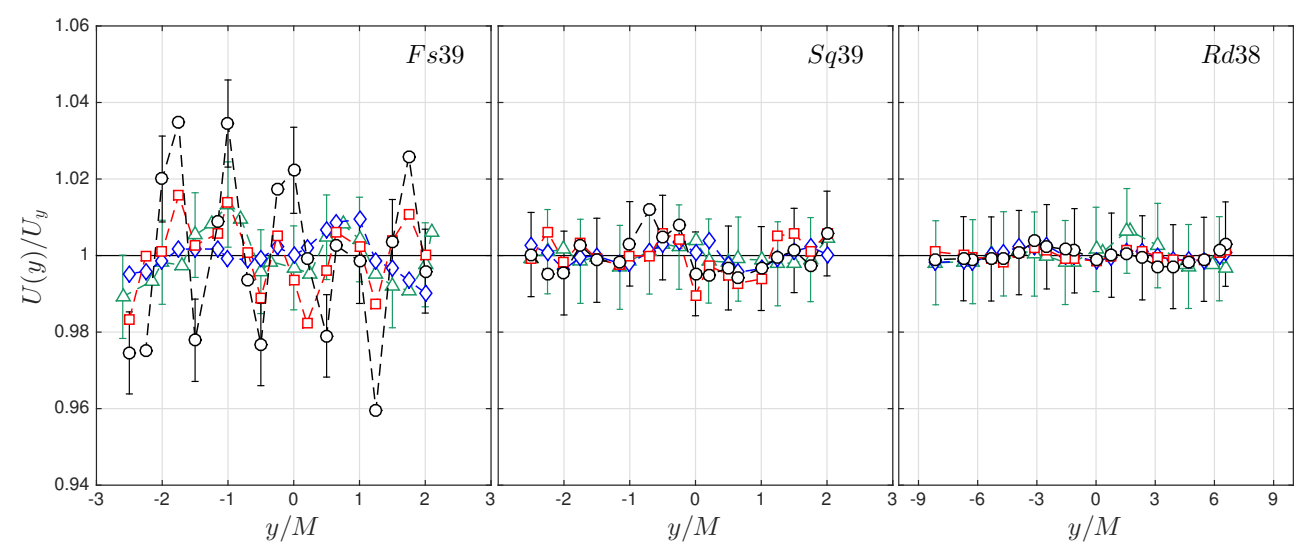

FIGURE 15. Transverse profiles of the normalised mean velocity for the conditions identified in table $3 ;(\bigcirc) x=1.25 \mathrm{~m},(\square) x=2.00 \mathrm{~m},(\diamond) x=3.25 \mathrm{~m},(\triangle) x=4.50 \mathrm{~m}$.

the turbulent kinetic energy equation, given by,

$$
\frac{U_{k}}{2} \frac{\partial\left\langle q^{2}\right\rangle}{\partial x_{k}}=-\left\langle u_{i} u_{j}\right\rangle \frac{\partial U_{i}}{\partial x_{j}}-\frac{\partial}{\partial x_{k}}\left(\frac{\left\langle u_{k} q^{2}\right\rangle}{2}\right)+\frac{\partial}{\partial x_{k}}\left(\frac{\left\langle u_{k} p\right\rangle}{\rho}\right)+\frac{\nu}{2} \frac{\partial^{2}\left\langle q^{2}\right\rangle}{\partial x_{m} \partial x_{m}}-\langle\epsilon\rangle,
$$

where Einstein's summation notation is used. In (A 1), the terms on the right-handside are production, triple-correlation transport, pressure transport, viscous diffusion, and dissipation, respectively. The left-hand-side represents the decay of turbulent kinetic energy. The terms of (A 1) that can be directly measured with the transverse scans are the production, specifically $\langle u v\rangle \partial U / \partial y$ and $\left\langle v^{2}\right\rangle \partial V / \partial y$, and the transverse transport of turbulent kinetic energy, $(\partial / \partial y)\left(\left\langle v q^{2}\right\rangle / 2\right)$. Hearst \& Lavoie (2014b) demonstrated that $\left\langle v^{2}\right\rangle \partial V / \partial y$ was much smaller than the other terms, which is consistent with the present measurements and hence this term is not shown here. Figures 16 and 17 show the relevant production and transverse transport terms for all three grids normalised by the mean dissipation across the span, $\langle\epsilon\rangle_{y}$. For $F s 39$, peaks in the production representing nearly $25 \%$ of the energy dissipation are present. Similarly, there are peaks of $\pm 20 \%$ of the dissipation in the transverse transport of turbulent kinetic energy generated in the wake of Fs39. These peaks decrease and become negligible as $x$ grows and the flow homogenizes, which is consistent with the results without a secondary contraction presented by Hearst \& Lavoie (2014b).

The first measurements of non-zero transverse transport in the wake of a fractal geometry were performed by Valente \& Vassilicos (2011) who remarked that similar measurements had never been performed in the wake of regular grids, and hence it was not known if transverse transport is indeed present there or not. For completeness, the production and transverse transport of turbulent kinetic energy for $S q 39$ and $R d 38$ are shown in figures 16 and 17 . It is evident that the regular grids do not have substantial production or transverse transport for measurements performed at the same streamwise positions as Fs39. However, we emphasize that the fractals also have diminishing production and transverse transport as their turbulence evolves downstream.

\section{REFERENCES}

Bailey, S. C. C., Kunkel, G. J., Hultmark, M., Vallikivi, M., Hill, J. P., Meyer, 


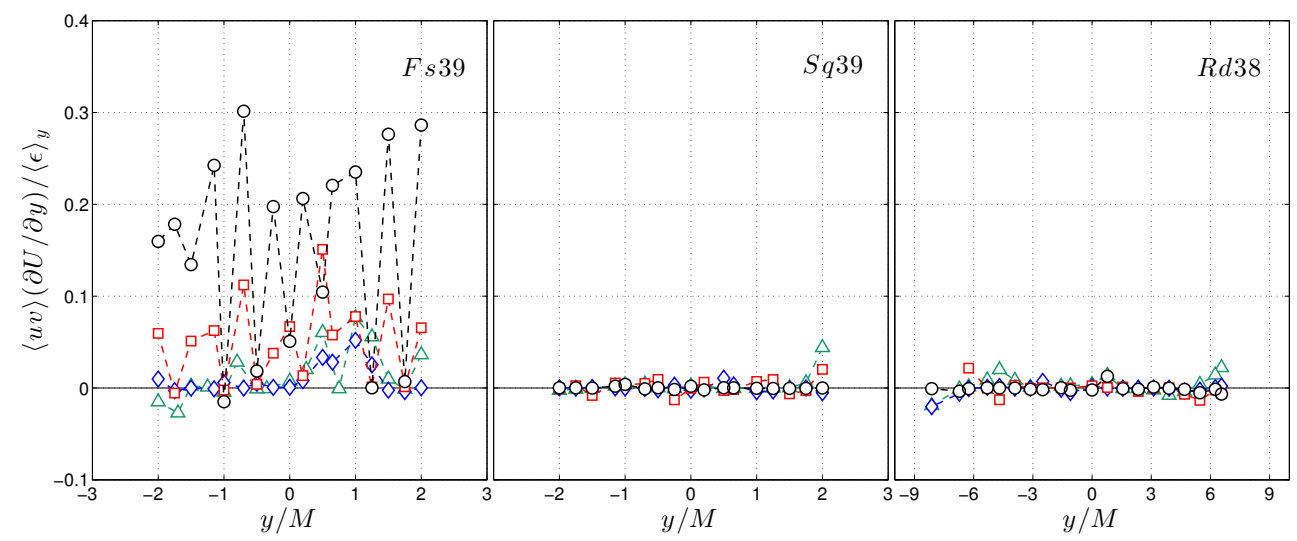

FiguRE 16. Transverse profiles of the production of turbulent kinetic energy for the conditions identified in table 3; $(\bigcirc) x=1.25 \mathrm{~m},(\square) x=2.00 \mathrm{~m},(\diamond) x=3.25 \mathrm{~m},(\triangle) x=4.50 \mathrm{~m}$. The estimated uncertainty on this quantity is on the order of $\pm 10 \%$, which is particularly related to the difficulty associated with determining the transverse gradient.

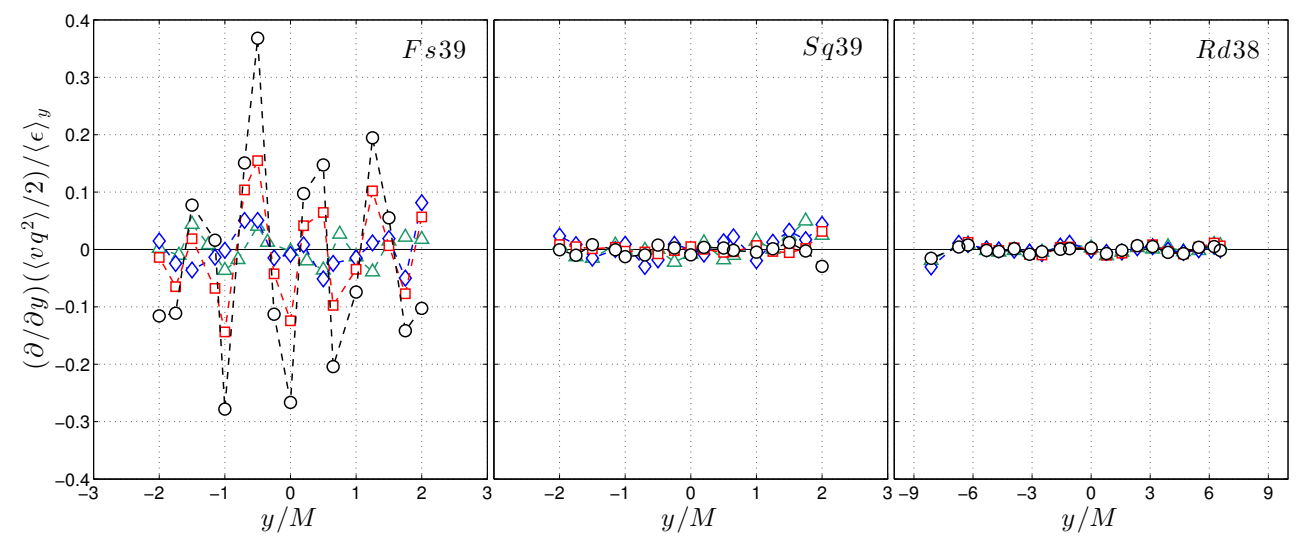

FiguRE 17. Transverse profiles of the transverse transport of turbulent kinetic energy for the conditions identified in table $3 ;(\bigcirc) x=1.25 \mathrm{~m},(\square) x=2.00 \mathrm{~m},(\diamond) x=3.25 \mathrm{~m},(\triangle) x=4.50 \mathrm{~m}$. The estimated uncertainty on this quantity is on the order of $\pm 12 \%$, which is particularly related to the difficulty associated with determining the transverse gradient.

K. A., Tsay, C., Arnold, C. B. \& Smits, A. J. 2010 Turbulence measurements using a nanoscale thermal anemometry probe. J. Fluid Mech. 663, 160-179.

Bell, J. H. \& Mehta, R. D. 1988 Contraction design for small low-speed wind tunnels. Tech. Rep.. NASA.

Benedict, L. H. \& Gould, R. D. 1996 Towards better uncertainty estimates for turbulence statistics. Exp. Fluids 22, 129-136.

Burattini, P. \& Antonia, R. A. 2005 The effect of different X-wire calibration schemes on some turbulence statistics. Exp. Fluids 38, 80-89.

Cafiero, G., Discetti, S. \& Astarita, T. 2015 Flow field topology of submerged jets with fractal generated turbulence. Phys. Fluids 27 (115103).

Castro, I.P. 2016 Dissipative distinctions. J. Fluid Mech. 788, 1-4.

Comte-Bellot, G. \& Corrsin, S. 1966 The use of a contraction to improve the isotropy of grid-generated turbulence. J. Fluid Mech. 25 (4), 657-682.

Dairay, T., Obligado, M. \& Vassilicos, J.C. 2015 Non-equilibrium scaling laws in axisymmetric turbulent wakes. J. Fluid Mech. 781, 166-195. 
Frisch, U. 1995 Turbulent Flows. Cambridge University Press.

Geipel, P., Goh, K.H.H. \& Lindstedt, R.P. 2010 Fractal-generated turbulence in opposed jet flows. Flow Turb. Comb. 85, 397-419.

Hearst, R. J., Buxton, O. R. H., Ganapathisubramani, B. \& Lavoie, P. 2012 Experimental estimation of fluctuating velocity and scalar gradients in turbulence. Exp. Fluids 53 (4), 925-942.

Hearst, R. J. \& Lavoie, P. $2014 a$ Decay of turbulence generated by a square-fractal-element grid. J. Fluid Mech. 741, 567-584.

Hearst, R. J. \& Lavoie, P. $2014 b$ Scale-by-scale energy budget in fractal element gridgenerated turbulence. J. Turb. 15 (8), 540-554.

Hearst, R. J. \& Lavoie, P. $2015 a$ The effect of active grid initial conditions on high Reynolds number turbulence. Exp. Fluids 56 (10), 185.

Hearst, R. J. \& Lavoie, P. $2015 b$ Velocity derivative skewness in fractal-generated, nonequilibrium grid turbulence. Phys. Fluids 27 (7), 071701.

Hurst, D. \& VAssilicos, J. C. 2007 Scalings and decay of fractal-generated turbulence. Phys. Fluids 19 (035103).

Isaza, J. C., Salazar, R. \& Warhaft, Z. 2014 On grid-generated turbulence in the nearand far field regions. J. Fluid Mech. 753, 402-426.

Jørgensen, F. E. 2002 How to measure turbulence with hot-wire anemometers - A practical guide. Dantec Dynamics.

Kinzel, M., Wolf, M., Holzner, M., Lüthi, B., Tropea, C. \& Kinzelbach, W. 2011 Simultaneous two-scale 3D-PTV measurements in turbulence under the influence of system rotation. Exp. Fluids 51, 75-82.

Kolmogorov, A. N. 1941 On the degeneration of isotropic turbulence in an incompressible viscous fluid. Dokl. Akad. Nauk SSSR 31, 538-541.

Kolmogorov, A. N. 1962 A refinement of previous hypotheses concerning the local structure of turbulence in a viscous incompressible fluid at high Reynolds number. J. Fluid Mech. 13, $82-85$.

Krogstad, P.-A. \& Davidson, P. A. 2010 Is grid turbulence Saffman turbulence? J. Fluid Mech. 642, 373-394.

Krogstad, P.-A. \& Davidson, P. A. 2011 Freely decaying, homogeneous turbulence generated by multi-scale grids. J. Fluid Mech. 680, 417-434.

Krogstad, P.-A. \& Davidson, P. A. 2012 Near-field investigation of turbulence produced by multi-scale grids. Phys. Fluids 24 (035103).

Laizet, S., Nedić, J. \& Vassilicos, J. C. 2015 The spatial origin of $-5 / 3$ spectra in gridgenerated turbulence. Phys. Fluids 27 (065115).

Laizet, S. \& VAssilicos, J. C. 2015 Stirring and scalar transfer by grid-generated turbulence in the presence of a mean scalar gradient. J. Fluid Mech. 764, 52-75.

Lavoie, P., Djenidi, L. \& Antonia, R. A. 2007 Effects of initial conditions in decaying turbulence generated by passive grids. J. Fluid Mech. 585, 395-420.

Lumley, J. L. 1992 Some comments on turbulence. Phys. Fluids A 4 (2), 203-211.

Mazellier, N. \& Vassilicos, J. C. 2010 Turbulence without Richardson-Kolmogorov cascade. Phys. Fluids 22 (075101).

Mi, J. \& Antonia, R. A. 2001 Effect of large-scale intermittency and mean shear on scalingrange exponents in a turbulent jet. Phys. Rev. E 64 (026302).

Mi, J., Deo, R. C. \& Nathan, G. J. 2005 Fast-convergence iterative scheme for filtering velocity signals and finding Kolmogorov scales. Phys. Rev. E 71 (066304).

Mohamed, M.S. \& LaRue, J.C. 1990 The decay power law in grid-generated turbulence. J. Fluid Mech. 219, 195-214.

Mydlarski, L. \& WARHAFt, Z. 1996 On the onset of high-Reynolds-number grid-generated wind tunnel turbulence. J. Fluid Mech. 320, 331-368.

Nagata, K., Sakai, Y., Inaba, T., Suzuki, H., Terashima, O. \& Suzuki, H. 2013 Turbulence structure and turbulence kinetic energy transport in multiscale/fractalgenerated turbulence. Phys. Fluids 25 (065102).

Nedić, J., Ganapathisubramani, B., Vassilicos, J. C., Borée, J., Brizzi, L. E. \& Spohn, A. 2012 Aeroacoustic performance of fractal spoilers. AIAA Journal 50 (12), 2695-2710. 
Nedić, J., Vassilicos, J.C. \& Ganapathisubramani, B. 2013 Axisymmetric turbulent wakes with new nonequilibrium similarity scalings. Phys. Rev. Lett. 111 (144503).

Saddoughi, S. G. \& Veeravalli, S. V. 1994 Local isotropy in turbulent boundary layers at high Reynolds number. J. Fluid Mech. 268, 333-372.

Seoud, R. E. \& Vassilicos, J. C. 2007 Dissipation and decay of fractal-generated turbulence. Phys. Fluids 19 (105108).

Sinhuber, M., Bodenschatz, E. \& Bewley, G. P. 2015 Decay of turbulence at high Reynolds numbers. Phys. Rev. Lett. 114 (034501).

Soulopoulus, N., Kerl, J., Sponfeldner, T., Beyrau, F., Hardalupas, Y., Taylor, A. M. K. P. \& VAssilicos, J. C. 2013 Turbulent premixed flames on fractal-grid-generated turbulence. Fluid Dyn. Res. 45 (6).

Thormann, A. \& Meneveau, C. 2014 Decay of homogeneous, nearly isotropic turbulence behind active fractal grids. Phys. Fluids 26 (025112).

Valente, P. C. \& Vassilicos, J. C. 2011 The decay of turbulence generated by a class of multiscale grids. J. Fluid Mech. 687, 300-340.

Valente, P. C. \& Vassilicos, J. C. 2012 Universal dissipation scaling for nonequilibrium turbulence. Phys. Rev. Lett. 108 (214503).

Valente, P. C. \& Vassilicos, J. C. 2014 The non-equilibrium region of grid-generated decaying turbulence. J. Fluid Mech. 744, 5-37.

Vallikivi, M., Hultmark, M., Bailey, S. C. C. \& Smits, A. J. 2011 Turbulence measurements in pipe flow using a nano-scale thermal anemometry probe. Exp. Fluids 51, 1521-1527.

Vassilicos, J. C. 2015 Dissipation in turbulent flows. Annu. Rev. Fluid Mech. 47, 95-114.

Verbeek, A. A., Bouten, T. W. F. M., Stoffels, G. G. M., Geurts, B. J. \& van der MeEr, T. H. 2015 Fractal turbulence enhancing low-swirl combustion. Comb. Flame 162, $129-143$.

Wang, H. \& George, W. K. 2002 The integral scale in homogeneous isotropic turbulence. J. Fluid Mech. 459, 429-443.

Weitemeyer, S., Reinke, N., Peinke, J. \& Hölling, M. 2013 Multi-scale generation of turbulence with fractal grids and an active grid. Fluid Dyn. Res. 45 (6), 061407.

WyngaARD, J. C. 1968 Measurements of small-scale turbulence structure with hot wires. J. Sci. Instr. 1 (2), 1105-1108.

Zhou, Y., Nagata, K., Sakai, Y., Suzuki, H., Ito, Y., Terashima, O. \& Hayase, T. 2014 Relevance of turbulence behind the single square grid to turbulence generated by regularand multiscale-grids. Phys. Fluids 26 (075105). 